\title{
BcMF30a and BcMF30c, Two Novel Non-Tandem CCCH Zinc-Finger Proteins, Function in Pollen Development and Pollen Germination in Brassica campestris ssp. chinensis
}

\author{
Liai Xu ${ }^{1,2} \mathbb{D}$, Xingpeng Xiong ${ }^{1,2}$, Weimiao Liu ${ }^{1,2}$, Tingting Liu ${ }^{1,2} \mathbb{D}$, Youjian Yu 3 \\ and Jiashu Cao ${ }^{1,2, *}$ \\ 1 Laboratory of Cell \& Molecular Biology, Institute of Vegetable Science, Zhejiang University, \\ Hangzhou 310058, China; 11416052@zju.edu.cn (L.X.); xiongxingpeng1989@163.com (X.X.); \\ 11616044@zju.edu.cn (W.L.); 11416009@zju.edu.cn (T.L.) \\ 2 Key Laboratory of Horticultural Plant Growth, Development and Quality Improvement, \\ Ministry of Agriculture/Zhejiang Provincial Key Laboratory of Horticultural Plant Integrative Biology, \\ Hangzhou 310058, China \\ 3 Department of Horticulture, College of Agriculture and Food Science, Zhejiang A \& F University, \\ Lin'an 311300, China; yjyu@zafu.edu.cn \\ * Correspondence: jshcao@zju.edu.cn; Tel.: +86-131-8501-1958
}

Received: 18 July 2020; Accepted: 31 August 2020; Published: 3 September 2020

\begin{abstract}
Chinese cabbage (Brassica campestris) is an economically important leaf vegetable crop worldwide. Mounting studies have shown that cysteine-cysteine-cysteine-histidine $(\mathrm{CCCH})$ zinc-finger protein genes are involved in various plant growth and development processes. However, research on the involvement of these genes in male reproductive development is still in its infancy. Here, we identified 11 male fertility-related $\mathrm{CCCH}$ genes in Chinese cabbage. Among them, a pair of paralogs encoding novel non-tandem $\mathrm{CCCH}$ zinc-finger proteins, Brassica campestris Male Fertility 30a (BcMF30a) and BcMF30c, were further characterized. They were highly expressed in pollen during microgametogenesis and continued to express in germinated pollen. Further analyses demonstrated that both BcMF30a and BcMF30c may play a dual role as transcription factors and RNA-binding proteins in plant cells. Functional analysis showed that partial bcmf30a bcmf30c pollen grains were aborted due to the degradation of pollen inclusion at the microgametogenesis phase, and the germination rate of viable pollen was also greatly reduced, indicating that BcMF30 $a$ and $B c M F 30 c$ are required for both pollen development and pollen germination. This research provided insights into the function of $\mathrm{CCCH}$ proteins in regulating male reproductive development and laid a theoretical basis for hybrid breeding of Chinese cabbage.
\end{abstract}

Keywords: $\mathrm{CCCH}$ zinc-finger protein; Brassica campestris ssp. chinensis; pollen development; pollen germination; male fertility

\section{Introduction}

The eukaryotic genome encodes a large number of proteins containing a variety of zinc-finger motifs and forms one of the most abundant protein families in eukaryotes. Based on the arrangements of cysteine (C) and/or histidine $(\mathrm{H})$ residues in the zinc-finger motif that bound to zinc ions, zinc-finger proteins can be classified into several different types, including $\mathrm{C}_{4}, \mathrm{C}_{6}, \mathrm{C}_{8}, \mathrm{C}_{2} \mathrm{H}_{2}, \mathrm{C}_{2} \mathrm{HC}, \mathrm{C}_{2} \mathrm{HC}_{5}, \mathrm{CCCH}, \mathrm{C}_{3} \mathrm{HC}_{4}$, and $\mathrm{C}_{4} \mathrm{HC}_{3}[1,2]$. These proteins are known to involve in numerous cellular functions (e.g., DNA recognition, RNA binding, transactivation, and protein-protein interaction) through their conserved zinc-finger motifs [3,4]. 
Cysteine-cysteine-cysteine-histidine ( $\mathrm{CCCH}$ ) zinc-finger proteins usually possess one to seven copies of $\mathrm{CCCH}$ motifs, which are newly defined as $\mathrm{C}-\mathrm{X}_{4-17}-\mathrm{C}-\mathrm{X}_{4-6}-\mathrm{C}-\mathrm{X}_{3}-\mathrm{H}$ [5-7]. $\mathrm{CCCH}$ genes are widely distributed in organisms from bacteria to eukaryotes [5,8], and members of this gene family in various plants have also been predicted in silico [5-7,9-12]. For instance, $68 \mathrm{CCCH}$-type zinc-finger protein genes divided into 11 subfamilies have been identified in the Arabidopsis thaliana genome [5]. The majority of these proteins contain one or two CCCH motifs. Notably, there are 11 members contain a tandem CCCH zinc-finger (TZF) motif preceded by a conserved arginine-rich (RR) region, known as AtTZF1-AtTZF11 [13-15].

In recent years, increasing reports have continuously demonstrated that $\mathrm{CCCH}$ genes play pivotal roles at different stages of plant growth and development. AtTZF6/PEI1 is the first CCCH gene to be functionally studied in plants, which is participated in embryo development [16]. AtTZF1 affects sugar-, ABA-, and GA-mediated growth [17]. AtTZF4/SOMNUS, AtTZF5, and AtTZF6/PEI1 play roles in seed germination [18,19]. AtC3H14 and AtC3H15/CALLOSE DEFECTIVE MICROSPORE 1 (CDM1) are involved in the regulation of secondary wall thickening and male fertility $[20,21]$. In rice (Oryza sativa), Delay of the Onset of Senescence (OsDOS), OsTZF1, GluB-1-binding Zinc Finger 1 (OsGZF1), ILA1 Interacting Protein 4 (OsIIP4), and Defective Callose in Meiosis 1 (DCM1) all function in plant development [22-26]. The $\mathrm{CCCH}$ proteins are also important regulators of stress responses. Several AtTZFs are shown to be triggered by various stimuli including salt stress, extreme temperatures, drought, nutritional deficiency, and hormone treatment $[5,18,27]$. Furthermore, the overexpression of these genes usually can enhance abiotic stress tolerance $[17,23,27,28]$. AtTZF9 is the only known RR-TZF gene that regulates immunity in Arabidopsis [29,30].

Nonetheless, $\mathrm{CCCH}$ proteins that have been functionally characterized mainly focus on model plants, Arabidopsis, and rice, and most of them are TZF proteins. To date, only a few $\mathrm{CCCH}$ genes have been characterized in other plants. For instance, the overexpression of cotton (Gossypium hirsutum) Zinc Finger Protein 1 (GhZFP1) can enhance tolerance to salt stress and resistance to fungal disease [31]. GhTZF1 is involved in drought stress responses and leaf senescence [32]. The pepper (Capsicum annuum L.) TZF $\mathrm{CaC} 3 \mathrm{H} 14$ acts as a positive regulator in response to Ralstonia solanacearum [33]. In poplar, two homologues of AtC3H14, PdC3H17 and PdC3H18, can also regulate secondary xylem formation [34]. For non-TZF proteins, functional studies are also very limited, even in model plants. HUA1, the only protein in Arabidopsis that contains six CCCH motifs, has been characterized as an RNA-binding protein and is involved in flower morphogenesis [35,36]. AtC3H17 plays multiple roles in plant development and stress response [37,38]. KHZ1 and KHZ2 could mediate flowering and senescence in Arabidopsis [39,40]. $\mathrm{DCM} 1$ is a five $\mathrm{CCCH}$ motif-containing protein in rice that is involved in male meiotic cytokinesis [26]. $\mathrm{IbC} 3 \mathrm{H} 18$ is a nuclear transcriptional activator that can enhance abiotic stress tolerance in sweet potato (Ipomoea batatas L.) [41]. Therefore, it seems that non-TZF proteins also play vital roles in plant growth and development, as well as stress response.

Chinese cabbage (Brassica campestris L. ssp. chinensis Makino) is a subspecies of B. campestris (AA, $2 n=20$ ) and one of the most important vegetable crops in Asia. In the breeding of Chinese cabbage, due to its strong heterosis characteristics, male sterile plants have been widely used in the production of hybrid seeds. The identification and functional analysis of genes related to male fertility is the basis of constructing male sterile lines. Although there is mounting evidence that $\mathrm{CCCH}$ genes are involved in various plant developments, only a handful of genes mentioned above have been characterized to play roles in male fertility. More than $100 \mathrm{CCCH}$ genes have been identified in silico in Chinese cabbage [11,12]. However, as far as we know, no function of any of these genes has been revealed, suggesting that their roles in male fertility are yet to be ascertained in Chinese cabbage.

Therefore, in this study, we screened several male fertility-related $\mathrm{CCCH}$ zinc-finger protein genes by identifying the differentially expressed genes between the ' $B$ cajh97-01A/B' genetic male sterile (GMS) A/B lines of B.campestris. Among them, both Brassica campestris Male Fertility 30a (BcMF30a) and its paralog $B c M F 30 c$ encode novel non-TZF proteins. The spatiotemporal expression profiles manifested that both of them were mainly expressed in developing pollen and geminated pollen. Further analyses demonstrated that both BcMF30a and BcMF30c have transcription activation activity 
in yeast and exhibit a dual nuclear and cytoplasmic localization; they contain RNA-binding domains as well, suggesting that they may play a dual role as transcription factors and RNA-binding proteins in plant cells. Moreover, we showed that mutation in BcMF30a and BcMF30c can cause abnormal pollen development, leading to pollen abortion and a failure of pollen germination. Together, our results indicate for the first time that two novel non-TZF proteins play key roles during microgametogenesis and pollen germination in Chinese cabbage.

\section{Results}

\subsection{Identification of Male Fertility-Related CCCH Zinc-Finger Protein Genes in B. campestris}

In long-term study, we generated and cultivated a stable Chinese cabbage-pak-choi 'Aijiaohuang' (Brassica campestris L. ssp. chinensis Makino) GMS A/B line system, which is named 'Bcajh97-01A/B'. The only difference between $\mathrm{A} / \mathrm{B}$ lines is that the male sterile plant ('Bcajh97-01A') undergoes an aberrant meiotic cytokinesis at the microsporogenesis stage and thus could not produce any functional mature pollen [42,43]. Therefore, we used the RNA-seq data of 'Bcajh97-01A/B' to identify the male fertility-related $\mathrm{CCCH}$ genes in B. campestris [44]. When analyzing the expression of these genes in five pollen development stages of ' $B$ cajh97-01A/B', 11 genes were significantly differentially expressed in at least at one stage between the fertile plant 'Bcajh97-01B' and the sterile plant 'Bcajh97-01A', and all genes were downregulated in the sterile plant except Bra007205 (Figure 1A). Moreover, the expression patterns of these genes were also different. More precisely, Bra001745/Bra038204 were nearly uniformly expressed at five different stages of floral buds; Bra004045 and Bra021817 were highly expressed at the mature pollen stage; Bra004288, Bra030149, Bra030151, and Bra032326 were tetrad stage specifically expressed genes; Bra015163/Bra013181 were mainly expressed during the bicellular pollen stage; and Bra007205 was a highly expressed gene at the pollen mother cell stage.

A

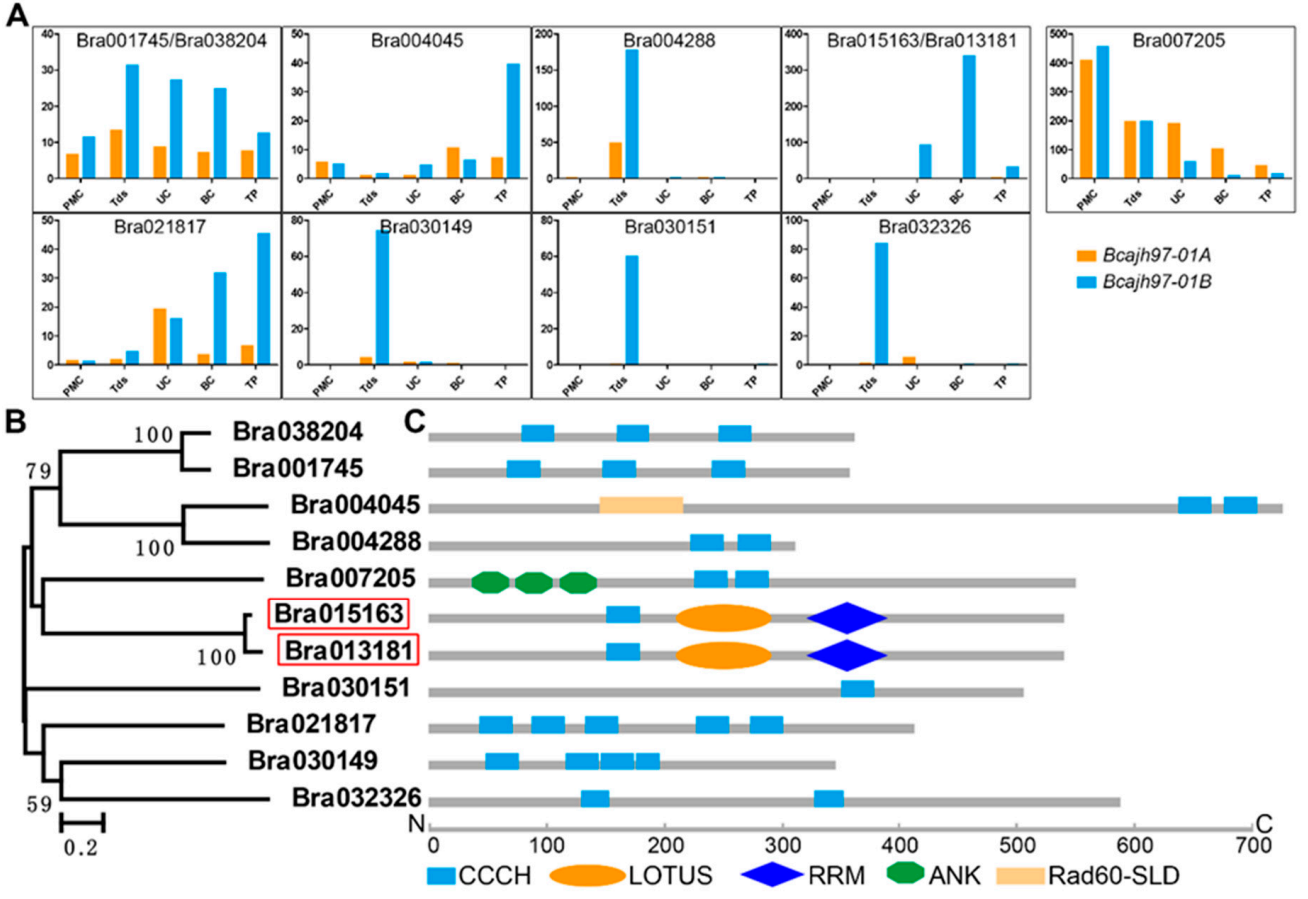

Figure 1. Identification of male fertility-related $\mathrm{CCCH}$ zinc-finger protein genes in B. campestris. (A) Expression analysis of $\mathrm{CCCH}$ zinc-finger protein genes in five pollen developmental stages of 'Bcajh97-01A/B' by RNA-seq. $\mathrm{PMC}$, pollen mother cell stage; Tds, tetrads stage; UC, unicellular pollen stage; $\mathrm{BC}$, bicellular pollen stage; $\mathrm{TP}$, tricellular pollen stage. (B) Phylogenesis analysis of 11 male fertility-related $\mathrm{CCCH}$ zinc-finger proteins. (C) Distribution of the conserved domains of male fertility-related CCCH zinc-finger proteins. 
Phylogenetic analysis showed that in addition to two pairs of paralogous genes (Bra001745/Bra038204 and Bra015163/Bra013181), Bra004045 and Bra004288 also have relatively close phylogenetic relationships (Figure 1B). The exon-intron structures of these 11 genes were also analyzed, and the number of exons varies greatly (Figure S1A). For example, Bra007205 has only 1 exon, while Bra032326 and Bra004045 have 11 and 12 exons, respectively. Protein domain analysis revealed that multiple proteins only contain $\mathrm{CCCH}$ motifs, ranging in number from 2 to 5 . However, Bra004045 also contains a Rad60-SLD domain, Bra007205 contains three ANK motifs, and both Bra015163 and Bra013181 contain a Limkain, Oskar, and TUdor-containing proteins 5 and 7 (LOTUS) domain and an RNA recognition motif (RRM) domain in addition to CCCH motif(s) (Figure 1C). Further exploration showed that most of the CCCH motifs of these 11 proteins are $\mathrm{C}-\mathrm{X}_{8}-\mathrm{C}-\mathrm{X}_{5}-\mathrm{C}-\mathrm{X}_{3}-\mathrm{H}$ type (Figure $\mathrm{S1B}$ ). The syntenic gene analysis indicated that most of these genes (except Bra030149) have syntenic paralogs in Arabidopsis thaliana, and the corresponding encoded proteins contain the same number of CCCH motifs except Bra004045 (Table S1). Unsurprisingly, there are also few studies on the function of these homologous genes in male reproductive growth.

Together, we identified $11 \mathrm{CCCH}$ zinc-finger protein genes that may be involved in male fertility in B. campestris. Although they are all expressed during pollen development, they display different expression patterns and differ in gene and protein structures.

\subsection{Expression Patterns of Two Novel Non-TZF Protein Genes, BcMF30a and BcMF30c}

Among the 11 male fertility-related genes identified above, two homologous genes, Bra015163 and Bra013181, encode proteins with only one $\mathrm{CCCH}$ motif, indicating that they are non-TZF protein genes. Bra015163 and Bra013181 are located in the less fractionized subgenome (LF) and the most fractionized subgenome (MF2), respectively, which means that the homologous gene in the moderate fractionized subgenome (MF1) has been lost. Thus, we named Bra015163 and Bra013181 as BcMF30a and BcMF30c, respectively. In addition to a CCCH motif, both BcMF30a and BcMF30c contain a LOTUS domain and an RRM domain (Figure 1C). Sequence analysis indicated that their amino acid similarity exceeded $90 \%$ (Figure S2).

To verify the effects of these two genes on Chinese cabbage, their spatiotemporal expressions were first analyzed with RT-PCR and qRT-PCR using total RNA extracted from various tissues. As shown in Figure 2A,B, both $B c M F 30 a$ and $B c M F 30 c$ transcripts only accumulated in inflorescence, and they were undetectable in root, stem, leaf, and silique. We also verified the expression of BcMF30a and $B c M F 30 c$ in the floral buds at different developmental stages of 'Bcajh97-01A/B'. Consistent with the expression profile based on RNA-seq analysis, they were mainly detected in 'Bcajh97-01B' floral buds at the bicellular pollen stage (Figure 2C,D). To investigate the expression of BcMF30 $a$ and BcMF30c in detail, we generated ProBcMF30a:GUS and ProBcMF30c:GUS transgenic Arabidopsis lines expressing the GUS ( $\beta$-glucuronidase) reporter gene under the control of the promoters of BcMF30a and BcMF30c, respectively. Similar to the above results, GUS staining can be detected in flowers at late developmental stages both in ProBcMF30a:GUS and ProBcMF30c:GUS transgenic plants, and GUS signal was mainly in anthers. In vitro pollen germination analysis revealed that ProBcMF30a:GUS and ProBcMF30c:GUS were also expressed in germinated pollen and pollen tubes (Figure 2E,F). These results indicated that $B c M F 30 a$ and $B c M F 30 c$ are pollen and pollen tube specifically expressed genes.

\subsection{Transcriptional-Activation Assay and Subcellular Localization of BcMF30a and BcMF30c}

Numerous zinc-finger proteins are known to bind to DNA through conserved zinc finger motifs and act as transcription factors to regulate the transcription of genes [3]. In order to verify whether BcMF30a and BcMF30c have transcript activation ability, yeast transactivation assay, a simple and quick method to test for transcriptional activation, was carried out. We fused the coding regions of BcMF30a and BcMF30c with the GAL4 DNA-binding domain in pGBKT7 vector (BD), respectively (Figure 3A). As shown in Figure 3B, the yeast cells harboring BD-AD, BD-BcMF30a, or BD-BcMF30c grew well on SD/-Trp-His-Ade medium, suggesting that both BcMF30a and BcMF30c possess transcriptional activation activity and might function as transcriptional activators, at least in yeast cells. 

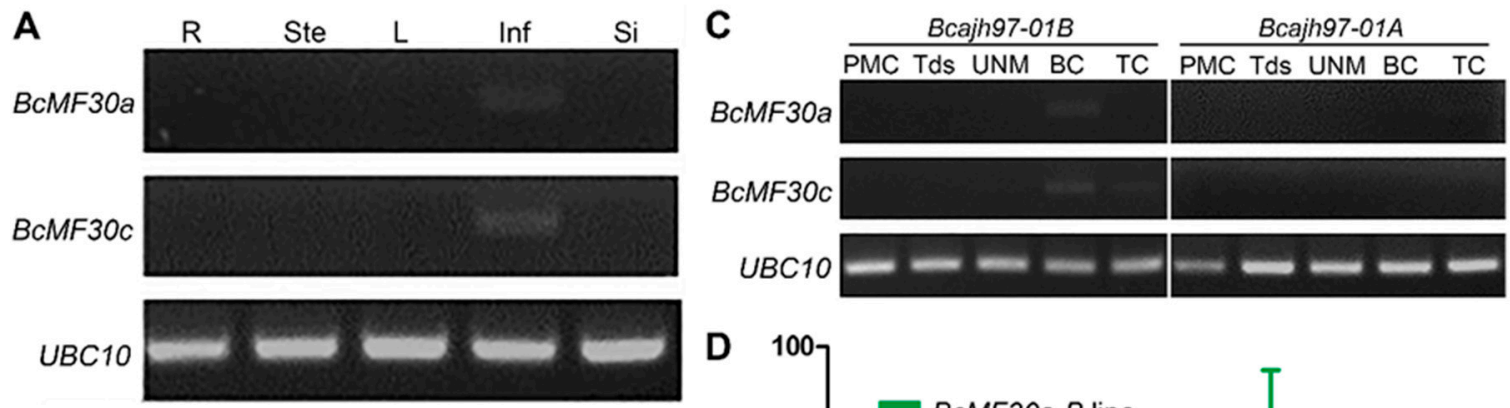

B
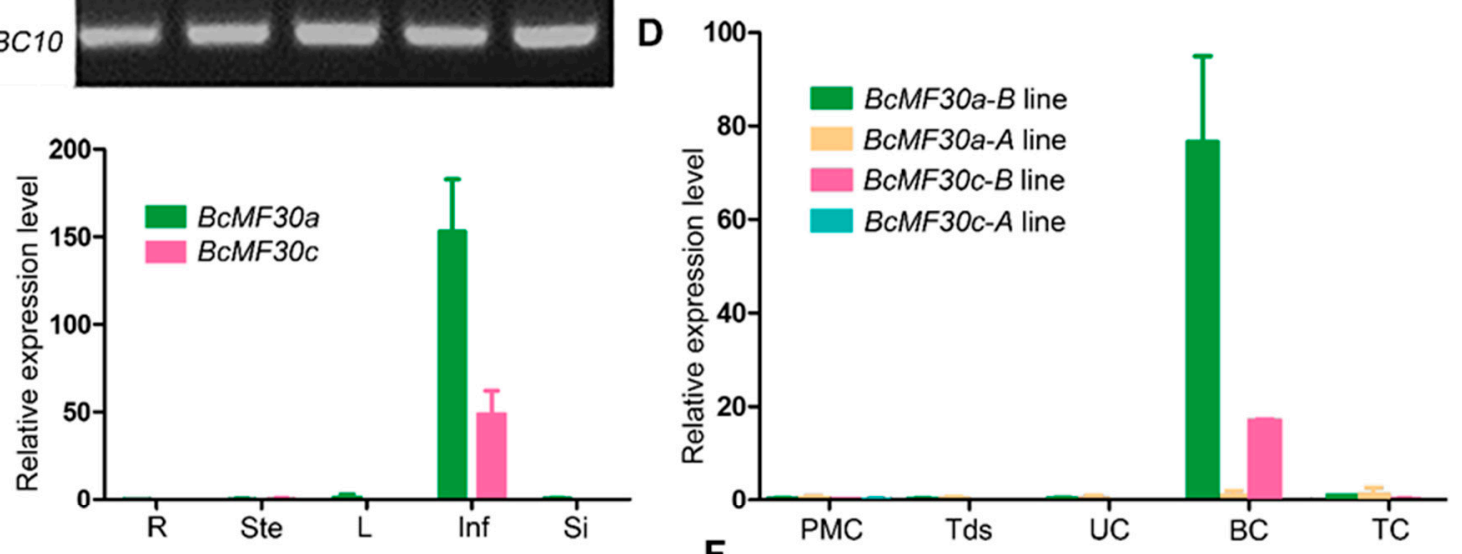

E
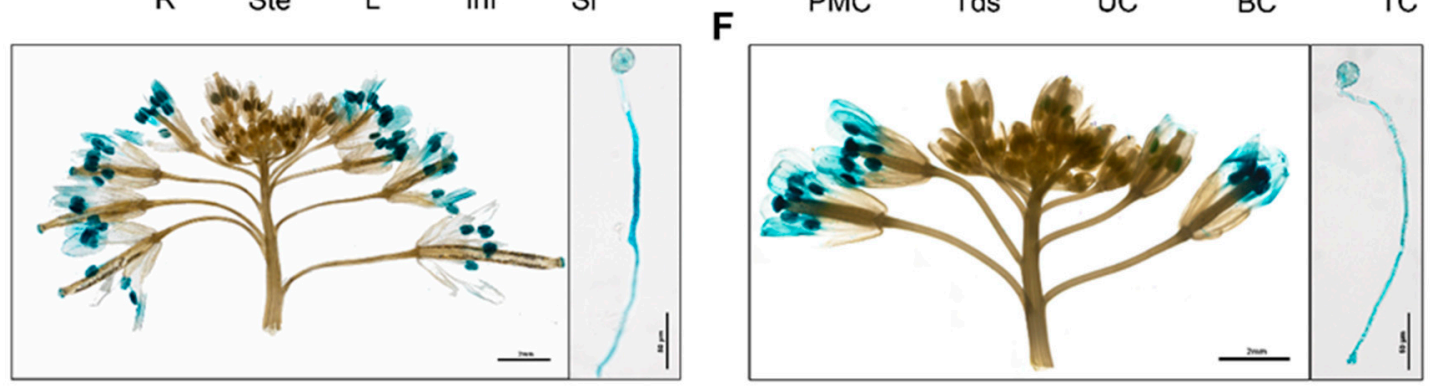

Figure 2. Expression analysis of $B c M F 30 a$ and $B c M F 30 c$. Semi-RT-PCR (A) and qRT-PCR (B) analyses of $B c M F 30 a$ and $B c M F 30 c$ in five major tissues of 'Bcajh97-01B'. R, root; Ste, stem; L, leaves; Inf, inflorescence; $\mathrm{Si}$, silique. Semi-RT-PCR (C) and qRT-PCR (D) analyses of BcMF30a and BcMF30c in floral buds of 'Bcajh97-01A/B' at different developmental stages. PMC, pollen mother cell stage; Tds, tetrads stage; $\mathrm{U}$, unicellular pollen stage; $\mathrm{BC}$, bicellular pollen stage; TC, tricellular pollen stage. Ubiquitously expressed UBC10 was used as internal control. Promoter-GUS activities of BcMF30a (E) and BcMF30c (F) in inflorescences and germinated pollen. Bars $=2 \mathrm{~mm}$ and $50 \mu \mathrm{m}$. GUS: $\beta$-glucuronidase.
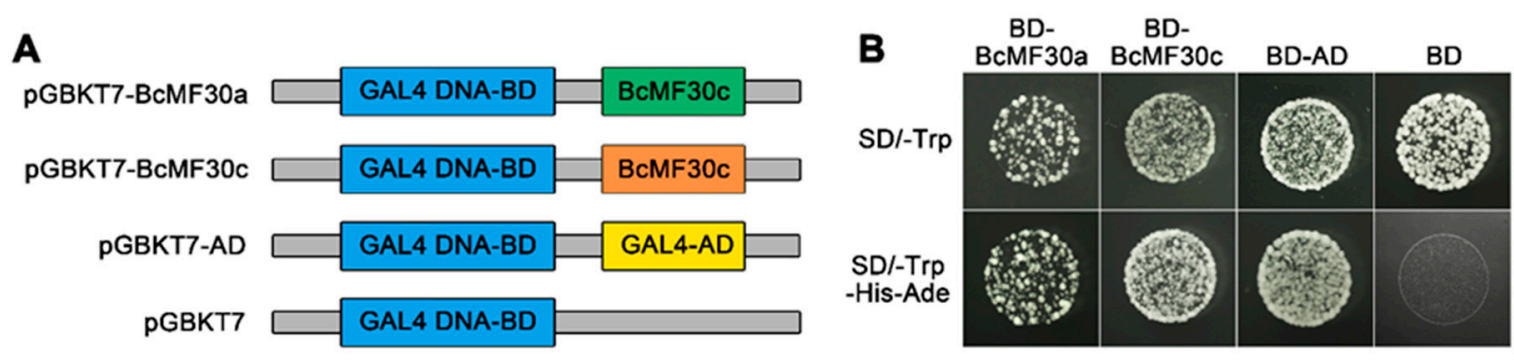

Figure 3. Transcriptional activation assays of BcMF30a and BcMF30c. (A) Schematic diagrams of plasmids used for transcriptional activation assays. GAL4 DNA-BD, GAL4 DNA-binding domain; GAL4-AD, GAL4 activation domain; BcMF30a and BcMF30c, the coding sequences of BcMF30a and BcMF30c. (B) The growth of transformed yeast on different yeast screening media.

To test whether BcMF30a and BcMF30c are nuclear proteins and thus similar to most typical transcription factors, we constructed fusion proteins of CaMV35S:eGFP-BcMF30a and CaMV35S:eGFP-BcMF30c (Figure 4A), which were transiently expressed in tobacco epidermal cells of H2B-RFP (nuclear marker) transgenic plants 
via Agrobacterium injection methodology. Surprisingly, in addition to the weak signal in the nucleus, we also observed that the fluorescence signals of eGFP-BcMF30a and eGFP-BcMF30c were distributed throughout the cytoplasm (Figure 4B). Similar subcellular localization patterns were also observed when enhanced green fluorescent protein (eGFP) was translationally fused to the C-terminus of BcMF30a and BcMF30c (Figure S3). These results indicated that BcMF30a and BcMF30c may not only function as transcription factors in the nucleus, but also perform unknown biological functions in the cytoplasm.

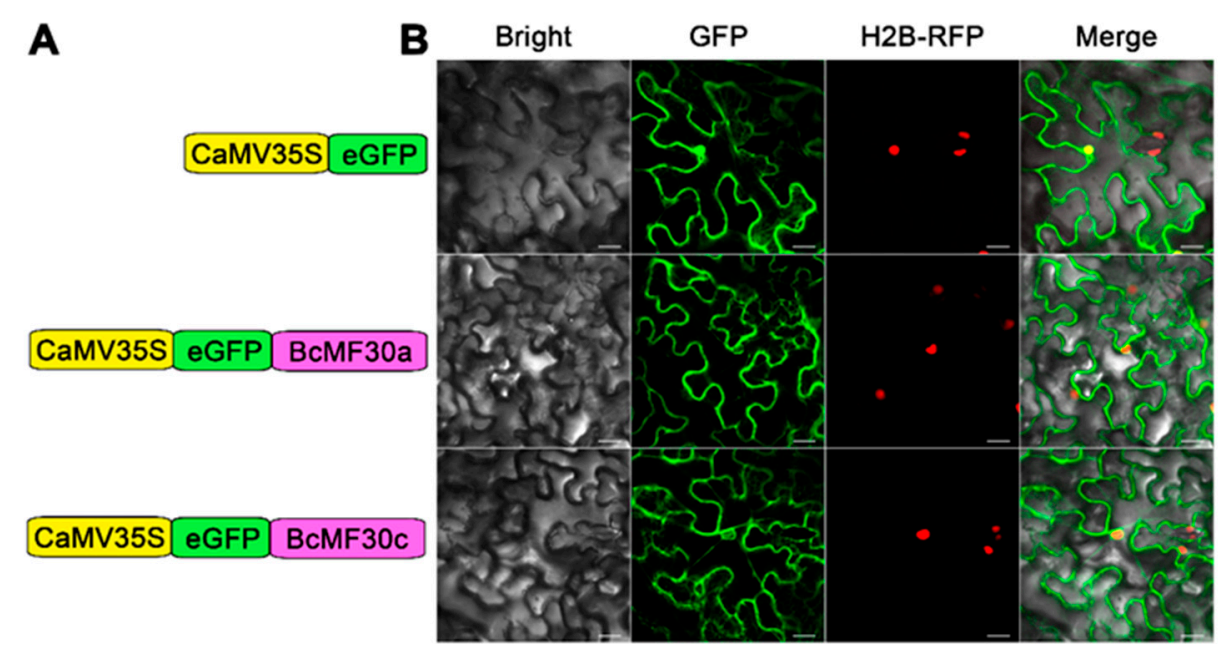

Figure 4. Subcellular localization of eGFP-BcMF30a and eGFP-BcMF30c fusion proteins in tobacco epidermal cells. (A) Constructs used for the subcellular localization analysis. (B) Both eGFP-BcMF30a and eGFP-BcMF30c were located in the nucleus and dispersed in the cytoplasm of tobacco epidermal cells. H2B is a marker protein of nucleus. Pictures represent white field images (Bright), epifluorescence (GFP and RFP) and merged images (Merge). Bar $=25 \mu \mathrm{m}$.

\subsection{Construction of Knockout Mutant of Chinese Cabbage Based on CRISPR/Cas9 System}

In order to explore the biological functions of $B c M F 30 a$ and $B c M F 30 c$, we planned to construct single-knockout and double-knockout mutants by using the complementation and clustered regularly interspaced short palindromic repeat/CRISPR-associated 9 (CRISPR/Cas9) system. Therefore, we designed a specific single-guide RNA (sgRNA) targeting BcMF30a (sgRNA-a) and BcMF30c (sgRNA-c) in the first exon, respectively. Meanwhile, we also designed an sgRNA targeting both BcMF30a and BcMF30c (sgRNA-ac) (Figure 5A). Then, we constructed CRISPR/Cas9 vectors using pBI121 (with ProAtUBQ:Cas9) and pCAMBIA1301 (with $2 \times$ CaMV35S:Cas9) as the vector backbones, respectively (both pBI121 and sgRNA-ac contain a Hind III cleavage site, so no CRISPR/Cas9 vector containing sgRNA-ac using pBI121 as the backbone has been constructed) (Figure S4). Multiple $\mathrm{T}_{0}$ transgenic lines were generated after introducing the constructs into Chinese cabbage calli (Figure S5 and Table S2).

Next, we examined the mutations by sequencing the PCR products amplified from the flanking regions of the target sites in all positive lines. We only detected gene-editing events in 6 of the 31 independent transgenic lines transformed with the pCA-sgRNA-ac vector (Table S2). The sequencing results showed that both $B c M F 30 a$ and $B c M F 30 c$ were edited in the five $\mathrm{T}_{0}$ lines (line ko-9, line ko-41, line ko-50, line ko-54, and line ko-64), and in line ko-58, only BcMF30c was edited. Further analysis showed that most $\mathrm{T}_{0}$ mutants were chimeras with 1-bp insertion (T/C/A in BcMF30a and T/G in $B c M F 30 c$ ) at exactly 3 bp upstream from the protospacer adjacent motif (PAM) (Figure 5B). The agarose gel electrophoresis results showed that one $\mathrm{T}_{0}$ line had a large fragment deletion in BcMF30a (line ko-64) and BcMF30c (line ko-58), respectively (Figure S6). Unfortunately, lines ko-9 and ko-58 failed to flower in the soil due to poor growth. Moreover, in $\mathrm{T}_{1}$ plants, mutations were only detected in the progeny of line ko-41, indicating that the mutations in other lines were not effectively inherited. Results of gene editing analysis showed that most of the $\mathrm{T}_{1}$ plants of line ko- 41 had biallelic mutations in both 
$B c M F 30 a$ and BcMF30c (Table S3), i.e., insert T or C before the PAM sites of BcMF30a, and insert T or G before the PAM sites of $B c M F 30 c$. Hereafter, these mutants are referred to as bcmf30a bcmf30c.

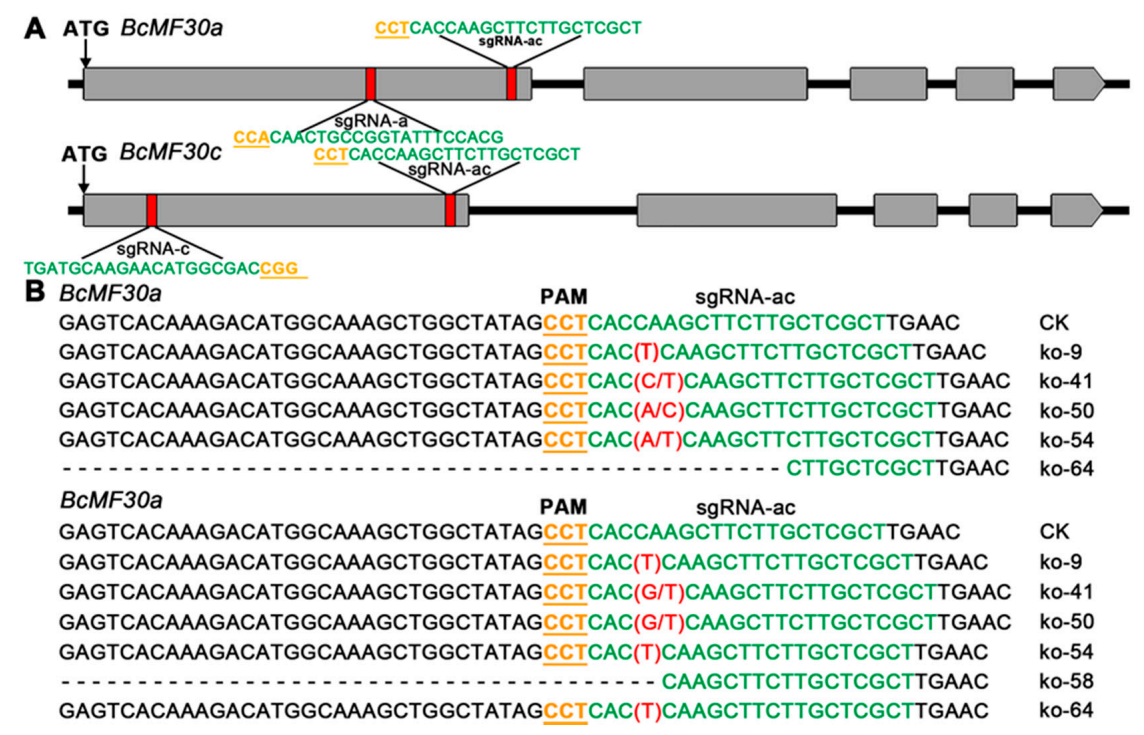

Figure 5. Construction of mutants by using the CRISPR/Cas9 system. (A) Genomic location of guide sequences (sgRNA-a, sgRNA-c, and sgRNA-ac) targeting BcMF30a and/or BcMF30c. (B) The gene editing of BcMF30a and BcMF30c in pCA-sgRNA-ac transgenic $\mathrm{T}_{0}$ lines. CRISPR/Cas9: complementation and clustered regularly interspaced short palindromic repeat/CRISPR-associated 9, sgRNA: single-guide RNA, sgRNA-a: sgRNA targeting BcMF30a, sgRNA-c: sgRNA targeting BcMF30c, sgRNA-ac: sgRNA targeting BcMF30a and BcMF30c.

Then, we predicted whether the mutated BcMF30 $a$ and $B c M F 30 c$ still have the potential to encode proteins. The results suggested that the mutated $B c M F 30 a$ and $B c M F 30 c$ may still encode the peptide chains, but their synthesis may be terminated prematurely, and it is predicted that the newly synthesized peptide chains lack the C-terminal of the full-length proteins containing the RRM domain (Figure 6A). Next, we performed qRT-PCR using RNA from $b c m f 30 a$ bcmf30c inflorescence to evaluate whether the mutated BcMF30a and BcMF30c can be transcribed. The results showed that transcripts can still be detected in bcmf30a bcmf30c, but the expression levels of most $\mathrm{T}_{1}$ plants were significantly lower than that of the control (Figure 6B).
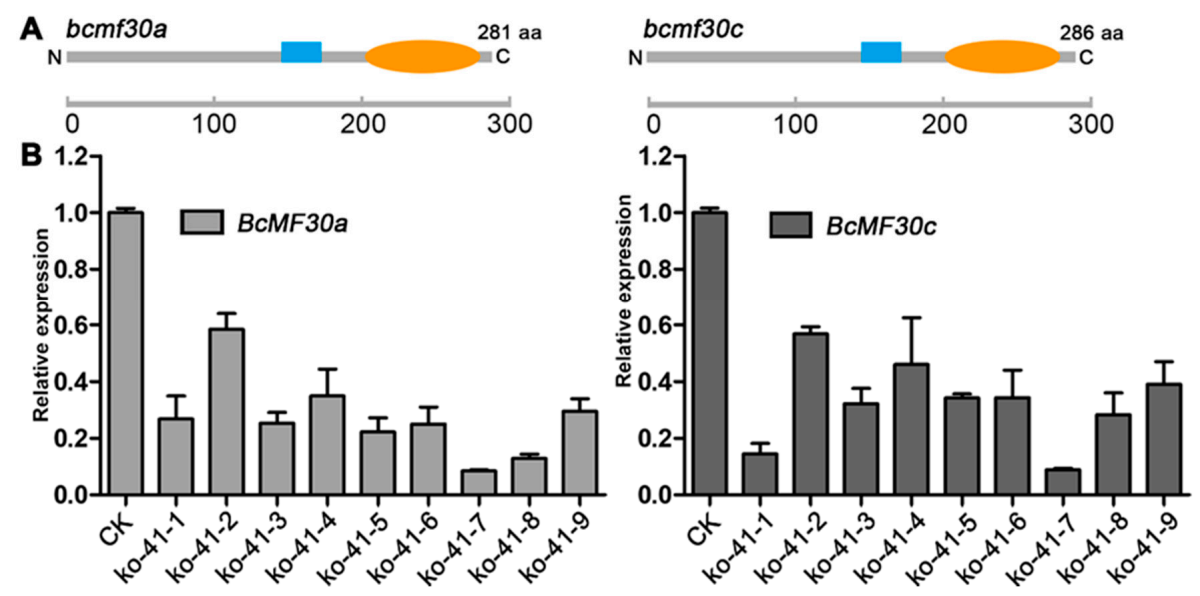

Figure 6. Quantitative detection and protein coding potential prediction of mutated $B c M F 30 a$ and mutated BcMF30c in bcmf30a bcmf30c mutants. (A) Prediction of proteins coded by the mutated BcMF30a $(b c m f 30 a)$ and mutated BcMF30c (bcmf30c). (B) The relative expression levels of $b c m f 30 a$ and $b c m f 30 c$ in the inflorescences of $b c m f 30 a b c m f 30 c$ by qRT-PCR analysis. 


\subsection{Mutations in BcMF30a and BcMF30c Do Not Affect Plant Vegetative Growth and Flower Formation}

Compared with control plants, the vegetative growth of all $\mathrm{T}_{0}$ lines and $b c m f 30 a$ bcmf30c plants did not show any significant differences (Figure S7A,B). Given that BcMF30 $a$ and BcMF30c were specifically expressed in inflorescence, we focused on whether the mutants exhibited defects during reproductive growth. The morphology and size of bcmf30a bcmf30c flowers were similar to those of the control plants. Further observation of the sepals, petals, stamens, and pistils also showed no evident abnormality (Figure S7C-P).

\section{6. bcmf30a bcmf30c Exhibits Partial Male Sterility}

To determine whether mutations in BcMF30a and BcMF30c cause abnormal pollen development, we conducted pollen viability testing and pollen morphology observation. The results of Alexander staining showed that on average, about $30.7 \%$ of pollen grains from $\mathrm{T}_{0}$ lines were aborted as compared with only $4.4 \%$ from the control plants (Figure 7A). Further analysis showed that up to $39.2 \%$ of $b c m f 30 a$ $b c m f 30 c$ mature pollen grains were nonviable (Figure 7B,C,H). Scanning electron microscopy (SEM) observation also demonstrated that partial mature pollen from $b c m f 30 a b c m f 30 c$ were shrunken and collapsed, but the other part of the mature pollen grains had no significant difference compared with the control pollen grains in morphology and size (Figure 7D,E,I,J). 4',6-diamidino-2-phenylindole (DAPI) staining showed that no nuclei were observed in aberrant pollen from bcmf30a bcmf30c, whereas normal-shaped pollen grains contain three nuclei (Figure 7F,G,K,L).
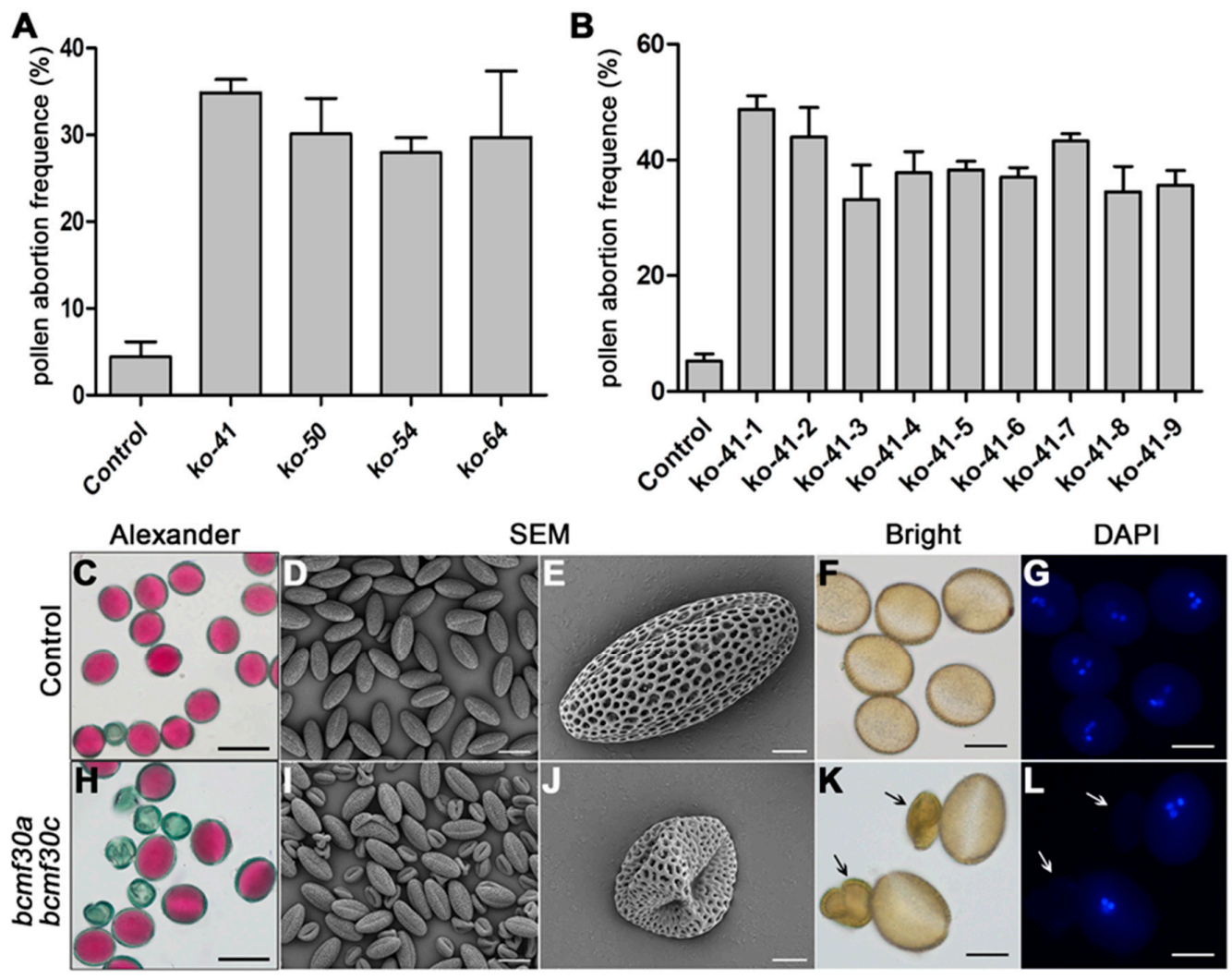

Figure 7. Cytological observation of mature pollen grains in bcmf30a bcmf30c mutants of B. campestris. Analysis of pollen abortion frequency in control, pCA-sgRNA-ac transgenic $\mathrm{T}_{0}$ lines (A), and $b c m f 30 a$ $b c m f 30 c$ mutants $(\mathbf{B})$. The values are the mean \pm SD. Alexander staining $(\mathbf{C}, \mathbf{H})$, SEM observation $(\mathbf{D}, \mathbf{E}, \mathbf{I}, \mathbf{J})$, and $4^{\prime}$,6-diamidino-2-phenylindole (DAPI) staining $(\mathbf{F}, \mathbf{G}, \mathbf{K}, \mathbf{L})$ of mature pollen grains in control plants and $b c m f 30 a b c m f 30 c$ mutants. Bars $=50 \mu \mathrm{m}$ in $(\mathbf{C}, \mathbf{H}), 30 \mu \mathrm{m}$ in $(\mathbf{D}, \mathbf{I}), 5 \mu \mathrm{m}$ in $(\mathbf{E}, \mathbf{J})$ and $20 \mu \mathrm{m}$ in $(\mathbf{F}, \mathbf{G}, \mathbf{K}, \mathbf{L})$. 
In order to clarify the anther development process in bcmf30a bcmf30c and to investigate the precise stage when pollen began to develop abnormally due to the mutations, semi-thin transverse sections were performed. No detectable differences between bcmf30a bcmf30c and the control pollen were observed inside the anther locules before the uninucleate stage (Figure 8A-C,F-H). Visible abnormalities appeared in the anthers of $b c m f 30 a b c m f 30 c$ at the bicellular stage, in which a large number of pollen grains were deformed and stained less (Figure $8 \mathrm{D}, \mathrm{I})$. By the tricellular stage, the pollen inclusion was almost completely degraded (Figure 8E,J).

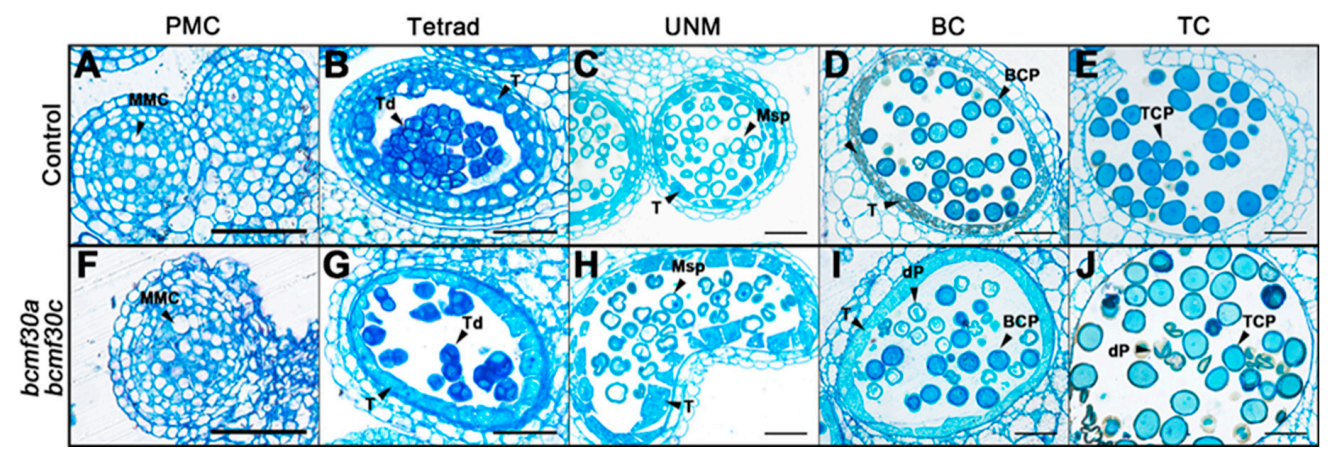

Figure 8. The semi-thin transverse sections of bcmf30a bcmf30c anthers. Semi-thin sections of anthers from the control plants (A-E) and bcmf30a bcmf30c (F-J) at the pollen mother cell stage (PMC, $(\mathbf{A}, \mathbf{F}))$, tetrad stage (Tetrad, $(\mathbf{B}, \mathbf{G}))$, uninucleate microspore stage $(\mathrm{UNM},(\mathbf{C}, \mathbf{H}))$, bicellular pollen stage $(\mathrm{BC},(\mathbf{D}, \mathbf{I}))$ and tricellular pollen stage (TC, (E,J)). BCP, bicellular pollen; MMC, microspore mother cell; Msp, microspore; P, pollen; $\mathrm{dP}$, degenerated pollen; $\mathrm{T}$, tapetum; TCP, tricellular pollen; Tds, tetrads. Bars $=25 \mu \mathrm{m}$.

The abnormalities of the $b c m f 30 a b c m f 30 c$ pollen grains were further determined by transmission electron microscopy (TEM). As shown in Figure 9, the nucleus in the control microspore was displaced to the side by a large vacuole at the late uninucleate stage (Figure 9A). However, during this stage, although the $b c m f 30 a b c m f 30 c$ microspores contain normally developed nuclei, most of the cytoplasm has been degraded (Figure 9D). By the bicellular stage, no nucleus was observed in bcmf30a bcmf30c pollen (Figure 9E). At the tricellular stage, mature control pollen grains were trinuclear with dense cytoplasm (Figure 9C). However, the degradation of microspores in bcmf30a bcmf30c plants resulted in aborted pollen that lacked any cytoplasmic components, leaving only the pollen wall that appeared to be intact (Figure 9F).

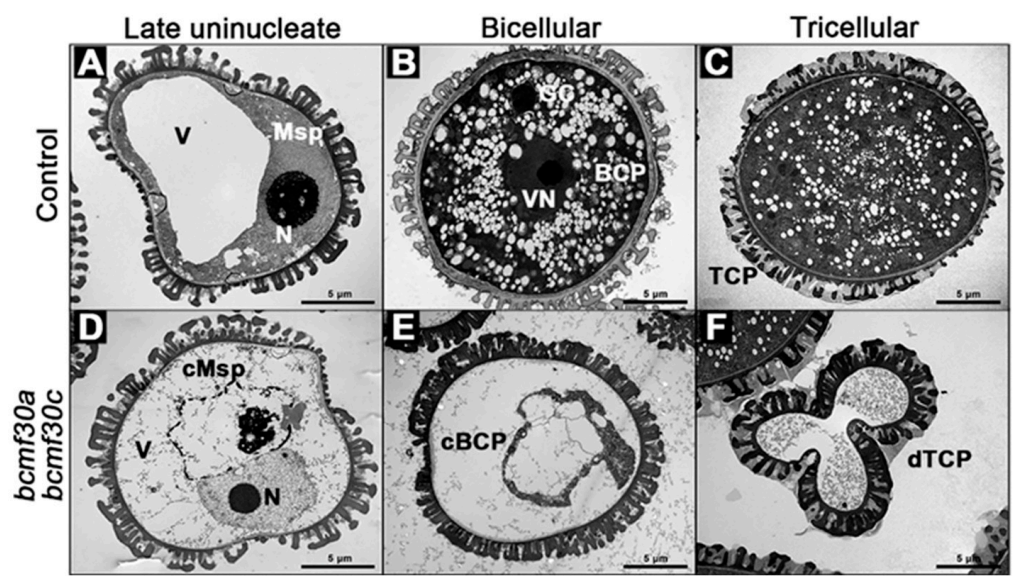

Figure 9. Transmission electron micrographs of pollen grains from bcmf30a bcmf30c mutants. Ultrastructure of pollen at different developmental stages from the control plants (A-C) and bcmf30a bcmf30c (D-G). (A,D) Late uninucleate stage; (B,E) bicellular pollen stage; $(\mathbf{C}, \mathrm{F}, \mathrm{G})$ tricellular pollen stage. $\mathrm{BCP}$, bicellular pollen; $\mathrm{CBCP}$, collapsed BCP; GC, generative cell; Msp, microspore; cMsp, collapsed Msp; N, nucleus; TCP, tricellular pollen; cTCP, collapsed TCP; dTCP, degenerated TCP; V, Vacule; VN, vegetative nucleus. Bars $=5 \mu \mathrm{m}$. 


\subsection{Pollen Germination Were Significantly Affected in bcmf30a bcmf30c}

To evaluate the effects of mutations in BcMF30a and BcMF30c on pollen germination, in vitro and in vivo pollen germination tests were conducted. In both control plants and bcmf30a bcmf30c plants, only viable pollen (identified by Alexander staining and morphological observation) was counted for pollen germination rate statistics. In vitro, less than half (39.2-49.4\%) of viable pollen grains from bcmf30a bcmf30c plants geminate normally, which is significantly lower than the germination rate in the control plants $(>80 \%$ ) (Figure 10A,B). The difference of pollen tube growth in pistils between the $b c m f 30 a$ bcmf30c plants and the control plants was also observed. The results revealed that the number of bcmf30a bcmf30c pollen tubes was much less than that of control pollen tubes, whether they were grown in the pistils of $b c m f 30 a$ bcmf30c plants or the control plants (Figure 10C). These results demonstrated that mutations in BcMF30a and BcMF30c not only affect pollen development, but also pollen germination.

A

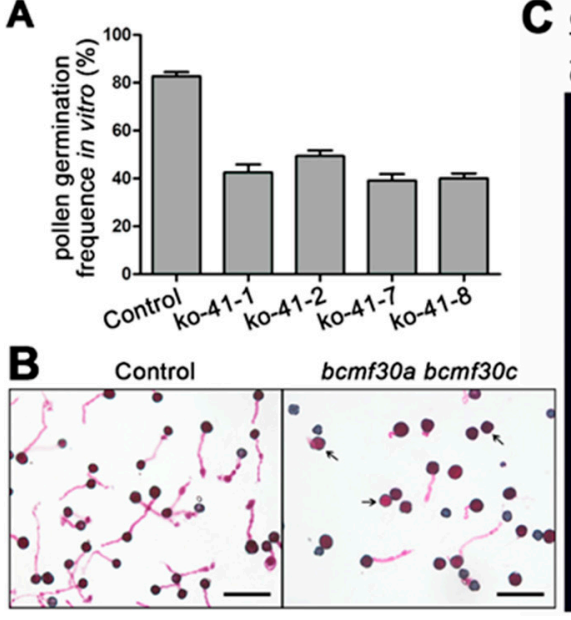

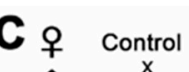
今 control bcmf30a bcmf30c bcmf30a bcmf $30 c$ $\begin{array}{cc}x & x \\ \text { bcmf30a bcmf30c } & \text { Control }\end{array}$
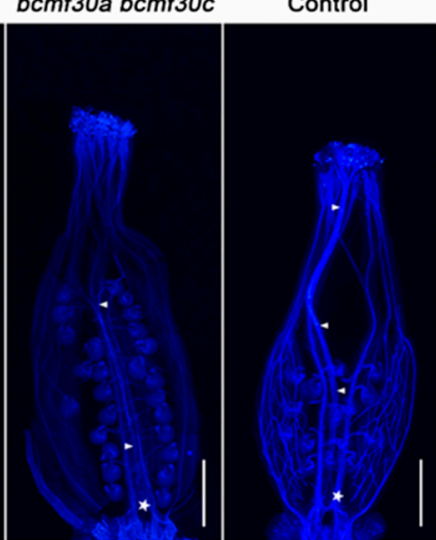

Control bcmf30a bcmf30c

Figure 10. The in vitro and in vivo germination tests of bcmf30a bcmf30c mature pollen. (A) Pollen germination frequencies of $b c m f 30 a b c m f 30 c$ pollen in vitro. Only viable pollen was counted for pollen germination rate statistics. The values are the mean $\pm \mathrm{SD}$. (B) Control and bcmf30a bcmf30c mature pollen germinated in vitro for $4 \mathrm{~h}$. (C) Aniline blue staining of pollen tubes in pistils after self-pollination and cross-pollination for $12 \mathrm{~h}$. The arrows indicated the pollen tubes and the asterisk indicated the end position of pollen tubes. Bars $=100 \mu \mathrm{m}$.

\section{Discussion}

Many species of Brassicaces are cultivated worldwide as oil seed or vegetable crops and display strong hybrid vigor or heterosis. Thus, hybrid breeding has become one of the main strategies for ensuring the production of Brassicaceae crops, including Chinese cabbage, which is one of the most important leafy vegetables in Asia. The identification of male fertility-related genes and the elucidation of the genetic, molecular, and biochemical mechanisms of male sterility caused by the functional loss of these genes are the top priority of both basic research and application of Chinese cabbage breeding. In this study, we identified 11 male fertility-related $\mathrm{CCCH}$ zinc-finger protein genes in Chinese cabbage and characterized the roles of two homologues, BcMF30 $a$ and BcMF30c, in pollen development and pollen germination for the first time. This work further enriched the functional studies of $\mathrm{CCCH}$ genes in plants, especially in male fertility.

\subsection{Male Fertility-Related CCCH Zinc-Finger Protein Genes in B. campestris}

As an important process during reproductive development, a large number of genes are expressed during pollen development, including the $\mathrm{CCCH}$ zinc-finger protein genes [45]. Recently, functional studies on some plant $\mathrm{CCCH}$ genes have been conducted, which demonstrated that these genes can act as vital 
regulators during both plant development and stress response, but much remains to be revealed. So far, only a handful of genes have been shown to be involved in pollen development. Moreover, these genes are mainly from model plants, including AtC3H14, AtC3H15/CDM1 in Arabidopsis, and DCM1 in rice $[20,21,26]$. In this study, we identified 11 male fertility-related $\mathrm{CCCH}$ zinc-finger protein genes in B. campestris. Surprisingly, among these 11 genes, three of them (Bra004045, BcMF30a, and BcMF30c) were identified as $\mathrm{CCCH}$ zinc-finger protein genes for the first time, which were not included in the 63 genes identified by Rameneni et al. (2018) [12] and the 103 genes identified by Pi et al. (2018) [11] as the $\mathrm{CCCH}$ zinc-finger protein genes in Brassica rapa. This result implies that the $\mathrm{CCCH}$ zinc-finger protein gene family in $B$. campestris contains a large number of members, and some members are likely to be missed when predicted in silico.

The expression profiles of some $\mathrm{CCCH}$ genes indicated that they may be involved in various abiotic stress responses, such as cold, drought, and salt stress responses [11,12]. To our knowledge, this is the first report linking the $\mathrm{CCCH}$ genes of Chinese cabbage to pollen development, and none of these 11 genes has been functionally studied. Among them, only one orthologous gene in Arabidopsis, AtC3H15/CDM1 (the homologous gene of Bra004288), has been revealed to be required for male fertility by regulating callose metabolism during microsporogenesis [20]. Here, we also found that Bra004288 was specifically expressed at the tetrad stage, which is a period when the newly formed microspores were blanketed and separated by a thick callose wall. Notably, there are three other CCCH genes (Bra030149, Bra030151, and Bra032326) specifically expressed by the tetrad (Figure 1A), suggesting that they may also be involved in the formation of tetrads. Several other genes exhibit different spatiotemporal expression patterns (Figure 1A), indicating that they may function at different pollen developmental stages. In this study, functional studies of $B c M F 30 a$ and $B c M F 30 c$, which began to be expressed at the uninucleate stage (Figure 2), confirmed that they are required for microgametogenesis. The regulatory functions of these male fertility-related $\mathrm{CCCH}$ genes in pollen development need further research.

\subsection{BcMF30a and BcMF30c, Two Novel Non-TZF Proteins, May Play a Dual Role in Plant Cells}

Based on the selected male fertility-related $\mathrm{CCCH}$ zinc-finger protein genes, we further isolated and characterized a pair of paralogous genes from Chinese cabbage flower buds, named BcMF30a and BcMF30c, which both encode novel non-TZF proteins with only one CCCH motif. As mentioned above, although two research studies have predicted the members of $\mathrm{CCCH}$ zinc-finger protein gene family in Brassica rapa [11,12], this study confirmed for the first time that BcMF30a and BcMF30c are also members of this family.

Similar to other types of zinc finger proteins that usually act as transcription factors, recent studies have shown that some $\mathrm{CCCH}$ zinc-finger proteins can bind to DNA and regulate gene expression at the transcriptional level. For example, the human $\mathrm{CCCH}$ zinc-finger protein, mini-chromosome maintenance 10 (Mcm10), is one of the DNA-replication factors that can serve as a physical link between the DNA polymerases and Mcm2-7 complex, and its central domain containing the $\mathrm{CCCH}$ motif can bind to DNA and several proteins [46]. Fetal liver zinc finger protein 1 (Fliz1), a zebrafish $\mathrm{CCCH}$ zinc-finger protein, acts as a transcriptional repressor that can specifically bind to the negative cis-acting element of GATA-3 [47]. In contrast, AtC3H17 in Arabidopsis and IbC3H18 in sweet potato both function as nuclear transcriptional activators [37,38]. Rice Leaf and tiller angle Increased Controller (OsLIC) and Arabidopsis AtC3H14 and AtC3H15/CDM1 can also bind to DNA and show transcriptional activity in yeast $[21,48]$. Here, we also found that BcMF30a and BcMF30c can exhibit transcriptional activation activity in yeast (Figure 3). Subcellular localization analysis showed that they can be localized to the nucleus, although the signals of both BcMF30a/BcMF30c-eGFP and eGFP-BcMF30a/BcMF30c fusion proteins in the nucleus were weaker than those of free-eGFP (Figure 4 and Figure S3). These findings demonstrated that BcMF30a and BcMF30c may function as nuclear transcriptional activators in plant cells. $\mathrm{IbC} 3 \mathrm{H} 18$ is the most likely homologous gene of $\mathrm{BcMF} 3 \mathrm{Oa}$ and BcMF30c in sweet potato. The study showed that the $303 \mathrm{C}$-terminal amino acid residues (containing an RRM domain) of IbC3H18 was responsible for its transcriptional activation activity [41]. 
Therefore, we speculate that the transcriptional activation domains of BcMF30a and BcMF30c probably are located at the $\mathrm{C}$-terminus of the proteins, which requires further research in the future.

Mounting research studies have shown that many identified $\mathrm{CCCH}$ zinc-finger proteins can also function as RNA-binding proteins and regulate gene expression at the post-transcriptional level. For instance, tristetraprolin (TTP), a mouse $\mathrm{CCCH}$ zinc-finger protein, can regulate mRNA turnover by specifically binding to the AU-rich elements in the $3^{\prime}$ untranslated regions of target mRNAs [49]. In Arabidopsis, HUA1 and Cleavage and Polyadenylation Specificity Factor 30 (AtCPSF30) are both RNA-binding proteins that can facilitate the maturation of the AGAMOUS pre-mRNA and regulate calmodulin-mediated RNA processing, respectively [36,50]. Several TZF proteins, such as AtTZF1, AtTZF9, AtC3H14, and AtC3H15/CDM1 in Arabidopsis, and OsTZF1 in rice, also show RNA-binding activity $[13,21,23,30]$. Unlike typical transcription factors that are usually localized only in the nucleus, in this study, we found that BcMF30a and BcMF30c have obvious signals in the cytoplasm in addition to the nucleus (Figure 4 and Figure S3), which suggests that they also play a role in the cytoplasm. In addition to containing a CCCH motif, BcMF30a and BcMF30c both contain a LOTUS domain and an RRM domain, both of which are putative RNA-binding domains [51-53], implying that they are likely to have RNA-binding activity. In fact, multiple CCCH zinc-finger proteins, such as AtTZF1, AtC3H14, and AtC3H15/CDM1 have been found to have a dual nuclear and cytoplasmic localization and can bind to DNA as well as RNA in vitro $[13,21]$. These results indicate that BcMF30a and BcMF30c may function as RNA-binding proteins in the cytoplasm.

Together, we believe that BcMF30a and BcMF30c are two novel non-TZF proteins in B. campestris, and they may play a dual role in plant cells, i.e., act as transcriptional activators in the nucleus and function as RNA-binding proteins in the cytoplasm. The next critical work toward understanding the molecular role of BcMF30a and BcMF30c in plant cells would be the identification of target genes transcriptionally activated by them and target RNAs that bind them.

\subsection{BcMF30a and BcMF30c Are Required for Microgametogenesis and Pollen Germination}

In flowering plants, the pollen grains produced during the reproductive growth stage harbor haploid male germ cells, which are vital for sexual reproduction. Pollen development takes place in the anther locules and can be divided into two major phases: microsporogenesis and microgametogenesis [54]. During microsporogenesis, the primary sporogenous cells produce the microsporocytes and then undergo meiosis to give rise to tetrads of haploid microspores enclosed and separated by thick callosal walls, which are subsequently released as individual microspores by callose enzyme [55]. During microgametogenesis, microspores successively undergo substantial cell growth, asymmetric division at pollen mitosis I (PMI), and germ cell division at PMII to form tricellular pollen, in which two sperm cells are engulfed by the vegetative cell [56]. Our expression analysis data showed that during pollen development, $B c M F 30 a$ and BcMF30c began to express in pollen at the microspore stage, peaked at the bicellular stage, and continued to express in mature pollen (Figures $1 \mathrm{~A}$ and 2). Then, we investigated whether pollen development in the bcmf30a bcmf30c mutant was affected. Our analyses showed that partial pollen were aborted in the mature pollen stage (Figure 7). Semi-thin sections and TEM results demonstrated that the $b c m f 30 a b c m f 30 c$ pollen developed normally during the microsporogenesis phase. However, the cytoplasm of microspores started to degrade at the late microspore stage, and it disappeared completely at the tricellular pollen stage, leaving only the pollen exine (Figures 8 and 9). Therefore, it is likely that the $b c m f 30 a b c m f 30 c$ mutation did not affect the formation of microspores, but it did disrupt the microgametogenesis.

To date, it is expected that near 100 genes affecting pollen development and cellular functions have been isolated in Arabidopsis by several genetic screening strategies [54,57]. The phenotypic analyses of certain gametophytic mutants indicate that some genes are specifically involved in cell division and patterning. For example, DUO POLLEN1 (DUO1) and its direct targets, DUO1-ACTIVATED ZINC FINGER PROTEIN1 (DAZ1) and DAZ2, form a DUO1-DAZ1/2 network model to control G2- to $\mathrm{M}$-phase transition and gamete differentiation [58,59]. However, mutations in most of other genes often 
lead to pollen degeneration and abortion at various developmental stages. For instance, mutations in Arabidopsis ABNORMAL GAMETOPHYTE (AGM), LONG-CHANIN BASE1 (LCB1)/LCB2A/LCB2C, and cellulose synthase genes (CESA1/2/3/6/9) can cause pollen abnormality at the microspore stage, bicellular stage, and tricellular stage, respectively [60-62]. Overall, proteins with a variety of functions are involved in various processes of pollen development, including chromatin organization, cell division and differentiation, cell wall construction, energy, metabolism, ion transport, and transcriptional regulation. As mentioned above, in view that both BcMF30a and BcMF30c may play a dual role in plant cells, we believe that they are involved in the microgametogenesis at both transcriptional and post-transcriptional levels. As for which biological processes are specifically affected, further research is urgently needed to clarify.

Pollen germination is also strictly regulated, which is a prerequisite for successful fertilization. At present, many genes essential for pollen germination have been identified, most of which are positive regulators, including NO POLLEN GERMINATION1 (NPG1) and NPG1-related genes, which encode $\mathrm{Ca}^{2+}$ sensor calmodulin (CaM)-binding proteins [63]. However, there are also a few negative regulators, such as JINGUBANG (JGB) and GTP-BINDING PROTEIN RELATED1 (GPR1), which can maintain the optimal pollen germination speed [64,65]. Here, we also observed the promoter activity of $B c M F 30 a$ and $B c M F 30 c$ in germinated pollen and pollen tubes (Figure 2E,F). Moreover, many viable bcmf30a $b c m f 30 c$ pollen grains cannot germinate normally both in vitro and in vivo (Figure 10). These findings suggest that $B c M F 30 a$ and $B c M F 30 c$ are also required for pollen germination and probably act as positive regulators. Notably, although this study only characterized the phenotype of $b c m f 30 a b c m f 30 c$ double mutants, given that BcMF30a and BcMF30c share high amino acid identity and have similar spatiotemporal expression patterns, we believe they are functional redundant.

Although the characterization of the $b c m f 30 a b c m f 30 c$ mutants provides insights into the role of the pollen-specific BcMF30a and BcMF30c genes, the molecular mechanisms of how they regulate pollen development and pollen germination and whether and how they cooperate to play roles of transcription factors and RNA-binding proteins to achieve transcriptional and post-transcriptional regulation remain largely unknown. Investigation of these issues will provide further insights into the molecular basis regulating male gametophyte development.

\section{Materials and Methods}

\subsection{Plant Material and Growth Conditions}

The B. campestris GMS A/B line system 'Bcajh97-01A/B' was planted in the experimental farm of Zhejiang University, Hangzhou, China. Brassica campestris L. ssp. chinensis var. parachinensis cv. Youqing 49 was used for the genetic transformation experiments due to its precocity characteristic. The transgenic Chinese cabbage of $\mathrm{T}_{0}$ lines, $\mathrm{T}_{1}$ plants, transgenic Arabidopsis thaliana Columbia- 0 plants, and H2B-RFP transgenic tobacco (Nicotiana benthamiana) plants were cultivated in a phytotron under long-day conditions $\left(22^{\circ} \mathrm{C}, 16 \mathrm{~h} \mathrm{light} / 18^{\circ} \mathrm{C}, 8 \mathrm{~h}\right.$ dark $)$.

\subsection{Expression Analysis of Male Fertility-Related CCCH Zinc-Finger Protein Genes in B. campestris}

In our previous study, we have obtained RNA-Seq data of 'Bcajh97-01A/B' at five typical flower bud development stages [44]. Since the only difference between the fertile line 'Bcajh97-01B' and sterile line 'Bcajh97-01A' is that 'Bcajh97-01A' is a male meiotic cytokinesis mutant that cannot produce any viable mature pollen grains, it is an ideal strategy to identify the $\mathrm{CCCH}$ zinc-finger protein genes that may be involved in male fertility by comparing the expression levels of these genes in 'Bcajh97-01A/B'. The data shown in Table S1 were collected from the TAIR (http://www.arabidopsis.org/) and Brassica (http://brassicadb.org/brad/) databases. Protein structure prediction was performed using SMART (http://smart.embl-heidelberg.de/). 


\subsection{RT-PCR and $q R T-P C R$}

Total RNAs were extracted by using RNAiso Plus (Takara, Shiga, Japan). Then, RNAs were transcribed into cDNAs with a PrimerScript RT reagent Kit (Takara, Shiga, Japan) and used as templates for RT-PCR and qRT-PCR analysis with specific primers (Table S4). UBC10 was used as the internal control. qRT-PCR was conducted by using a SYBR ${ }^{\circledR}$ Premix Ex Taq ${ }^{\mathrm{TM}}$ Kit (Takara, Japan) on a CFX96 Real-Time System (Bio-Rad, Hercules, CA, USA). Results were calculated using the $2^{-\Delta \Delta C t}$ method. Three technical repeats were performed.

\section{4. $\beta$-Glucuronidase (GUS) Histochemical Staining Assay}

The promoter sequences upstream of the ATG codons of BcMF30a (1720-bp) and BcMF30c (1919-bp) were amplified with gene-specific primers (Table S4) and cloned into pBI101 vector flanking the GUS reporter gene to create the fusion constructs, ProBcMF30a:GUS and ProBcMF30c:GUS. The floral-dip method mediated by Agrobacterium was used to transfer plasmids into Arabidopsis plants [66]. Transgenic plants were screened in MS agar plates containing kanamycin. GUS staining of inflorescences and germinated pollens were performed as described by Mudunkothge et al. (2014) [67].

\subsection{Transcriptional Activation Assay in Yeast}

The full lengths of $B c M F 30 a$ and $B c M F 30 c$ coding sequences were obtained by PCR with specific primers and cloned into the $p G B K-T 7$ vector. Then, the $p B D-B c M F 30 a, p B D-B c M F 30 c, p B D-A D$ (positive control), and $p B D-G A L 4$ (negative control) plasmids were transfected into the yeast strain (AH109). The transformed yeast cells were grown on SD/-Trp and SD/-Trp/-His/-Ade plates (lacking threonine, histidine, and adenine) at $30^{\circ} \mathrm{C}$ for 3 days. The transcriptional activation activities of BcMF30a and $B c M F 30 c$ were evaluated according the growth status of yeast cells on screening plates. The primers used are listed in Table S4.

\subsection{Subcellular Localization}

To observe the subcellular localization of $B c M F 30 a$ and $B c M F 30 c$, we amplified the coding sequences with specific primers (Table S4) and then inserted into the $p F G C-e G F P$ vector to form $p F G C-B c M F 30 a / c-e G F P$ and $p F G C-e G F P-B c M F 30 a / c$. Then, the fusion vectors were transiently transformed into 4-week-old H2B-RFP transgenic tobacco leaves by an infiltrated method. Fluorescent signals were analyzed 2 days after transformation under a laser confocal scanning microscope (Nikon, A1, Tokyo, Japan).

\subsection{Construction of CRISPR/Cas9 Vectors and Generation of Mutants}

For each BcMF30a and BcMF30c targeting site, two complementary oligonucleotides with 20-bp target sequences (sgRNA-a, sgRNA-c, and sgRNA-ac) were synthesized (Table S4). Each set of oligo pairs was annealed to generate double-stranded DNA with 4-bp overhangs on both ends, which were cloned into the Bbs I site of vectors containing the $p A t U 6-26$ :sgRNA-scaffold. According to the procedures shown in Figure S4, the CRISPR/Cas9 vectors targeting BcMF30a (pBI-sgRNA-a, pCA-sgRNA-a), $B c M F 30 c$ (pBI-sgRNA-c, pCA-sgRNA-c), and targeting both BcMF30a and BcMF30c (pCA-sgRNA-ac) were constructed. Then, all CRISPR/Cas9 vectors were transformed into the fertile Chinese cabbage (Youqing 49) by an Agrobacterium-mediated transformation method as described previously [68]. The DNA of generated $\mathrm{T}_{0}$ lines was extracted and then analyzed by a PCR screening approach with specific primers. For all the positive transgenic lines, the PCR products amplified from the flanking regions of the target sites were sequenced to determine mutations in BcMF30a and BcMF30c. For $\mathrm{T}_{0}$ line plants with mutations, the $T_{1}$ plants were propagated. Then, gene mutations in $T_{1}$ plants were also confirmed by PCR and sequencing. The expression of mutated BcMF30a and BcMF30c were analyzed by qRT-PCR according to the method mentioned above. The primers used are listed in Table S4. 


\subsection{Phenotypic and Cytological Observation of Pollen and Pollen Germination Assays}

When performing phenotypic and cytological observations, tissue-cultured plants without transgenic events were used as controls for $\mathrm{T}_{0}$ lines, and plants grown from seeds were used as controls for $b c m f 30 a$ bcmf30c plants. The mature pollen grains were dyed with Alexander stain [69] to investigate the pollen viability. DAPI solution [70] was used to detect the nuclei in pollen grains. The stained pollen grains were observed and photographed under a fluorescent microscope (Nikon, ECLIPSE 90i, Tokyo, Japan). For SEM, pollen grains were smeared on SEM carriers, and then the sample was coated with gold-palladium in Hitachi MC1000 ion sputter for about $10 \mathrm{~min}$. The images were observed and captured with a microscope (GeminiSEM 300, Carl Zeiss Microscopy GmbH, Oberkochen, Germany). TEM analyses as well as in vitro and in vivo pollen germination tests were conducted as described by Lin et al. (2014) [71] with some modifications. The samples for TEM observation were also used for semi-thin transverse sections observation. The detailed procedures referred to Lin et al. (2018) [72].

\section{Conclusions}

Collectively, we identified 11 male fertility-related $\mathrm{CCCH}$ zinc-finger protein genes in $B$. campestris for the first time. Moreover, we further isolated and characterized two non-TZF genes, BcMF30 $a$ and $B c M F 30 c$, which were specifically expressed in pollen during microgametogenesis and germinated pollen and pollen tubes. Molecular function analysis indicated that both BcMF30a and BcMF30c may play a dual role as transcription factors and RNA-binding proteins in plant cells. Knockout of BcMF30a and BcMF30c by using CRISPR/Cas9 technology results in partial pollen abortion and a significant reduction in pollen germination. These results indicate that BcMF30a and BcMF30c play important roles in the pollen development and pollen germination of Chinese cabbage, and they are likely to function at both the transcriptional and post-transcriptional regulatory pathways.

Supplementary Materials: Supplementary Materials can be found at http://www.mdpi.com/1422-0067/21/17/ 6428/s1. Table S1. Detailed information of male fertility-related CCCH zinc-finger genes in Brassica campestris and information relevant to Arabidopsis thaliana. Table S2. $\mathrm{T}_{0}$ lines and gene editing of plants transformed by CRISPR-Cas9 vectors. Table S3. Genotypes of 9 bcmf30a bcmf30c plants generated by line ko-41. Table S4. Sequence of primers used in this study. Figure S1. The exon-intron configurations of 11 male fertility-related CCCH zinc-finger protein genes in Brassica campestris. Figure S2. Sequence alignment of BcMF30a and BcMF30c. Figure S3. Subcellular localization of BcMF30a-eGFP and BcMF30c-eGFP fusion proteins in tobacco epidermal cells. Figure S4. Flow chart of constructing CRISPR-Cas9 knockout vectors. Figure S5. PCR analyses of transgenic $\mathrm{T}_{0}$ lines transformed with CRISPR/Cas9 knockout vectors of BcMF30a and BcMF30c. Figure S6. PCR analyses of BcMF30a and $B c M F 30 c$ in pCA-sgRNA-ac transgenic $\mathrm{T}_{0}$ lines. Figure S7. Morphological observation of plants and floral organs of bcmf30a bcmf30c plants.

Author Contributions: Conceptualization, L.X. and J.C.; methodology, L.X., X.X.; software, L.X., W.L.; validation, L.X., X.X. and T.L.; formal analysis, L.X., W.L.; investigation, L.X., T.L.; resources, J.C.; data curation, L.X.; writing-original draft preparation, L.X.; writing-review and editing, Y.Y., J.C.; visualization, L.X.; supervision, J.C.; project administration, L.X., J.C.; funding acquisition, J.C. All authors have read and agreed to the published version of the manuscript.

Funding: This research was funded by grant from the National Natural Science Foundation of China (No. 31772311).

Conflicts of Interest: The authors declare no conflict of interest.

\section{Abbreviations}

\begin{tabular}{|c|c|}
\hline $\mathrm{ABA}$ & abscisic acid \\
\hline $\mathrm{bp}$ & base pair \\
\hline GA & gibberellin \\
\hline CaMV & cauliflower mosaic virus \\
\hline $\mathrm{CCCH}$ & Cysteine-cysteine-cysteine-histidine \\
\hline CRISPR/Cas9 & $\begin{array}{l}\text { complementation and clustered regularly interspaced short palindromic } \\
\text { repeat/CRISPR-associated } 9\end{array}$ \\
\hline GFP & green fluorescent protein \\
\hline GMS & genetic male sterile \\
\hline GUS & $\beta$-glucuronidase \\
\hline
\end{tabular}




$\begin{array}{ll}\text { LOTUS } & \text { Limkain, Oskar, and TUdor-containing proteins } 5 \text { and } 7 \\ \text { PAM } & \text { protospacer adjacent motif } \\ \text { PCR } & \text { polymerase chain reaction } \\ \text { PMI } & \text { pollen mitosis I } \\ \text { PMII } & \text { pollen mitosis II } \\ \text { Pre-mRNA } & \text { precursor messenger RNA } \\ \text { qRT-PCR } & \text { quantitative reverse transcriptase polymerase chain reaction } \\ \text { RNA-seq } & \text { RNA sequencing } \\ \text { RRM } & \text { RNA recognition motif } \\ \text { SEM } & \text { scanning electron microscopy } \\ \text { TEM } & \text { transmission electron microscopy } \\ \text { TZF } & \text { tandem CCCH zinc-finger }\end{array}$

\section{References}

1. Takatsuji, H. Zinc-finger proteins: The classical zinc finger emerges in contemporary plant science. Plant Mol. Biol. 1999, 39, 1073-1078. [CrossRef] [PubMed]

2. Ciftci-Yilmaz, S.; Mittler, R. The zinc finger network of plants. Cell. Mol. Life Sci. 2008, 65, 1150-1160. [CrossRef] [PubMed]

3. Laity, J.H.; Lee, B.M.; Wright, P.E. Zinc finger proteins: New insights into structural and functional diversity. Curr. Opin. Struct. Biol. 2001, 11, 39-46. [CrossRef]

4. Hall, T.M.T. Multiple modes of RNA recognition by zinc finger proteins. Curr. Opin. Struct. Biol. 2005, 15, 367-373. [CrossRef] [PubMed]

5. Wang, D.; Guo, Y.; Wu, C.; Yang, G.; Li, Y.; Zheng, C. Genome-wide analysis of CCCH zinc finger family in Arabidopsis and rice. BMC Genom. 2008, 9, 44. [CrossRef] [PubMed]

6. Peng, X.; Zhao, Y.; Cao, J.; Zhang, W.; Jiang, H.; Li, X.; Ma, Q.; Zhu, S.; Cheng, B. CCCH-type zinc finger family in maize: Genome-wide identification, classification and expression profiling under abscisic acid and drought treatments. PLOS ONE 2012, 7, e40120. [CrossRef]

7. Yuan, S.; Xu, B.; Zhang, J.; Xie, Z.; Cheng, Q.; Yang, Z.; Cai, Q.; Huang, B. Comprehensive analysis of CCCH-type zinc finger family genes facilitates functional gene discovery and reflects recent allopolyploidization event in tetraploid switchgrass. BMC Genom. 2015, 16, 129. [CrossRef]

8. Kramer, S.; Kimblin, N.C.; Carrington, M. Genome-wide in silico screen for CCCH-type zinc finger proteins of Trypanosoma brucei, Trypanosoma cruzi and Leishmania major. BMC Genom. 2010, 11, 283. [CrossRef]

9. $\mathrm{Xu}, \mathrm{R}$. Genome-wide analysis and identification of stress-responsive genes of the $\mathrm{CCCH}$ zinc finger family in Solanum lycopersicum. Mol. Genet. Genom. 2014, 289, 965-979. [CrossRef]

10. Pradhan, S.; Kant, C.; Verma, S.; Bhatia, S. Genome-wide analysis of the CCCH zinc finger family identifies tissue specific and stress responsive candidates in chickpea (Cicer arietinum L.). PLoS ONE 2017, 12, e0180469. [CrossRef]

11. Pi, B.; He, X.; Ruan, Y.; Jang, J.-C.; Huang, Y. Genome-wide analysis and stress-responsive expression of $\mathrm{CCCH}$ zinc finger family genes in Brassica rapa. BMC Plant Biol. 2018, 18, 373. [CrossRef]

12. Rameneni, J.J.; Dhandapani, V.; Paul, P.; Devaraj, S.P.; Choi, S.R.; Yi, S.Y.; Kim, M.-S.; Hong, S.; Oh, S.H.; Oh, M.-H.; et al. Comprehensive analysis of $\mathrm{CCCH}$ zinc-finger-type transcription factors in the Brassica rapa genome. Hortic. Environ. Biotechnol. 2018, 59, 729-747. [CrossRef]

13. Pomeranz, M.C.; Hah, C.; Lin, P.-C.; Kang, S.G.; Finer, J.J.; Blackshear, P.J.; Jang, J.-C. The Arabidopsis tandem zinc finger protein AtTZF1 traffics between the nucleus and cytoplasmic foci and binds both DNA and RNA. Plant Physiol. 2010, 152, 151-165. [CrossRef]

14. Bogamuwa, S.P.; Jang, J.-C. Tandem CCCH zinc finger proteins in plant growth, development and stress response. Plant Cell Physiol. 2014, 55, 1367-1375. [CrossRef]

15. Jang, J.-C. Arginine-rich motif-tandem $\mathrm{CCCH}$ zinc finger proteins in plant stress responses and post-transcriptional regulation of gene expression. Plant Sci. 2016, 252, 118-124. [CrossRef]

16. Li, Z; Thomas, T.L. PEI1, an embryo-specific zinc finger protein gene required for heart-stage embryo formation in Arabidopsis. Plant Cell 1998, 10, 383-398. [CrossRef] 
17. Lin, P.-C.; Pomeranz, M.C.; Jikumaru, Y.; Kang, S.G.; Hah, C.; Fujioka, S.; Kamiya, Y.; Jang, J.-C. The Arabidopsis tandem zinc finger protein AtTZF1 affects ABA- and GA-mediated growth, stress and gene expression responses. Plant J. 2011, 65, 253-268. [CrossRef]

18. Bogamuwa, S.; Jang, J.-C. The Arabidopsis tandem CCCH zinc finger proteins AtTZF4, 5 and 6 are involved in light-, abscisic acid- and gibberellic acid-mediated regulation of seed germination. Plant Cell Environ. 2013, 36, 1507-1519. [CrossRef]

19. Yang, W.; Chen, Z.; Huang, Y.; Chang, G.; Li, P.; Wei, J.; Yuan, X.; Huang, J.; Hu, X. Powerdress as the novel regulator enhances Arabidopsis seeds germination tolerance to high temperature stress by histone modification of SOM locus. Plant Sci. 2019, 284, 91-98. [CrossRef]

20. Lu, P.; Chai, M.; Yang, J.; Ning, G.; Wang, G.; Ma, H. The Arabidopsis CALLOSE DEFECTIVE MICROSPORE1 gene is required for male fertility through regulating callose metabolism during microsporogenesis. Plant Physiol. 2014, 164, 1893-1904. [CrossRef]

21. Chai, G.; Kong, Y.; Zhu, M.; Yu, L.; Qi, G.; Tang, X.; Wang, Z.; Cao, Y.; Yu, C.; Zhou, G. Arabidopsis C3H14 and $\mathrm{C} 3 \mathrm{H} 15$ have overlapping roles in the regulation of secondary wall thickening and anther development. J. Exp. Bot. 2015, 66, 2595-2609. [CrossRef]

22. Kong, Z.; Li, M.; Yang, W.; Xu, W.; Xue, Y. A novel nuclear-localized CCCH-type zinc finger protein, OsDOS, is involved in delaying leaf senescence in rice. Plant Physiol. 2006, 141, 1376-1388. [CrossRef]

23. Jan, A.; Maruyama, K.; Todaka, D.; Kidokoro, S.; Abo, M.; Yoshimura, E.; Shinozaki, K.; Nakashima, K.; Yamaguchi-Shinozaki, K. OsTZF1, a CCCH-tandem zinc finger protein, confers delayed senescence and stress tolerance in rice by regulating stress-related genes. Plant Physiol. 2013, 161, 1202-1216. [CrossRef]

24. Chen, Y.; Sun, A.; Wang, M.; Zhu, Z.; Ouwerkerk, P.B.F. Functions of the CCCH type zinc finger protein OsGZF1 in regulation of the seed storage protein GluB-1 from rice. Plant Mol. Biol. 2014, 84, 621-634. [CrossRef]

25. Zhang, D.; Xu, Z.; Cao, S.; Chen, K.; Li, S.; Liu, X.; Gao, C.; Zhang, B.; Zhou, Y. An uncanonical CCCH-tandem zinc-finger protein represses secondary wall synthesis and controls mechanical strength in rice. Mol. Plant. 2018, 11, 163-174. [CrossRef]

26. Zhang, C.; Shen, Y.; Tang, D.; Shi, W.; Zhang, D.; Du, G.; Zhou, Y.; Liang, G.; Li, Y.; Cheng, Z. The zinc finger protein DCM1 is required for male meiotic cytokinesis by preserving callose in rice. PLoS Genet. 2018, 14, e1007769. [CrossRef]

27. Lee, S.-j.; Jung, H.J.; Kang, H.; Kim, S.Y. Arabidopsis zinc finger proteins AtC3H49/AtTZF3 and AtC3H20/AtTZF2 are involved in ABA and JA responses. Plant Cell Physiol. 2012, 53, 673-686. [CrossRef]

28. Wang, W.; Liu, B.; Xu, M.; Jamil, M.; Wang, G. ABA-induced CCCH tandem zinc finger protein OsC3H47 decreases ABA sensitivity and promotes drought tolerance in Oryza sativa. Biochem. Biophys. Res. Commun. 2015, 464, 33-37. [CrossRef]

29. Maldonado-Bonilla, L.D.; Eschen-Lippold, L.; Gago-Zachert, S.; Tabassum, N.; Bauer, N.; Scheel, D.; Lee, J. The Arabidopsis tandem zinc finger 9 protein binds RNA and mediates pathogen-associated molecular pattern-triggered immune responses. Plant Cell Physiol. 2014, 55, 412-425. [CrossRef]

30. Tabassum, N.; Eschen-Lippold, L.; Athmer, B.; Baruah, M.; Brode, M.; Maldonado-Bonilla, L.D.; Hoehenwarter, W.; Hause, G.; Scheel, D.; Lee, J. Phosphorylation-dependent control of an RNA granule-localized protein that fine-tunes defence gene expression at a post-transcriptional level. Plant J. 2020, 101, 1023-1039. [CrossRef]

31. Guo, Y.-H.; Yu, Y.-P.; Wang, D.; Wu, C.-A.; Yang, G.-D.; Huang, J.-G.; Zheng, C.-C. GhZFP1, a novel $\mathrm{CCCH}$-type zinc finger protein from cotton, enhances salt stress tolerance and fungal disease resistance in transgenic tobacco by interacting with GZIRD21A and GZIPR5. New Phytol. 2009, 183, 62-75. [CrossRef]

32. Zhou, T.; Yang, X.; Wang, L.; Xu, J.; Zhang, X. GhTZF1 regulates drought stress responses and delays leaf senescence by inhibiting reactive oxygen species accumulation in transgenic Arabidopsis. Plant Mol. Biol. 2014, 85, 163-177. [CrossRef] [PubMed]

33. Qiu, A.; Lei, Y.; Yang, S.; Wu, J.; Li, J.; Bao, B.; Cai, Y.; Wang, S.; Lin, J.; Wang, Y.; et al. CaC3H14 encoding a tandem $\mathrm{CCCH}$ zinc finger protein is directly targeted by CaWRKY40 and positively regulates the response of pepper to inoculation by Ralstonia solanacearum. Mol. Plant Pathol. 2018, 19, 2221-2235. [CrossRef] [PubMed]

34. Chai, G.; Qi, G.; Cao, Y.; Wang, Z.; Yu, L.; Tang, X.; Yu, Y.; Wang, D.; Kong, Y.; Zhou, G. Poplar PdC3H17 and PdC3H18 are direct targets of PdMYB3 and PdMYB21, and positively regulate secondary wall formation in Arabidopsis and poplar. New Phytol. 2014, 203, 520-534. [CrossRef] [PubMed] 
35. Li, J.; Jia, D.; Chen, X. HUA1, a Regulator of Stamen and Carpel Identities in Arabidopsis, Codes for a Nuclear RNA Binding Protein. Plant Cell 2001, 13, 2269-2281. [CrossRef] [PubMed]

36. Rodríguez-Cazorla, E.; Ripoll, J.J.; Andújar, A.; Bailey, L.J.; Martínez-Laborda, A.; Yanofsky, M.F.; Vera, A. K-homology nuclear ribonucleoproteins regulate floral organ identity and determinacy in Arabidopsis. PLoS Genet. 2015, 11, e1004983. [CrossRef]

37. Seok, H.-Y.; Woo, D.-H.; Park, H.-Y.; Lee, S.-Y.; Tran, H.T.; Lee, E.-H.; Vu Nguyen, L.; Moon, Y.-H. AtC3H17, a non-tandem $\mathrm{CCCH}$ zinc finger protein, functions as a nuclear transcriptional activator and has pleiotropic effects on vegetative development, flowering and seed development in Arabidopsis. Plant Cell Physiol. 2016, 57, 603-615. [CrossRef]

38. Seok, H.-Y.; Nguyen, L.V.; Park, H.-Y.; Tarte, V.N.; Ha, J.; Lee, S.-Y.; Moon, Y.-H. Arabidopsis non-TZF gene AtC3H17 functions as a positive regulator in salt stress response. Biochem. Biophys. Res. Commun. 2018, 498, 954-959. [CrossRef]

39. Yan, Z.; Jia, J.; Yan, X.; Shi, H.; Han, Y. Arabidopsis KHZ1 and KHZ2, two novel non-tandem CCCH zinc-finger and K-homolog domain proteins, have redundant roles in the regulation of flowering and senescence. Plant Mol. Biol. 2017, 95, 549-565. [CrossRef]

40. Yan, Z.; Shi, H.; Liu, Y.; Jing, M.; Han, Y. KHZ1 and KHZ2, novel members of the autonomous pathway, repress the splicing efficiency of FLC pre-mRNA in Arabidopsis. J. Exp. Bot. 2020, 71, 1375-1386. [CrossRef]

41. Zhang, H.; Gao, X.; Zhi, Y.; Li, X.; Zhang, Q.; Niu, J.; Wang, J.; Zhai, H.; Zhao, N.; Li, J.; et al. A non-tandem $\mathrm{CCCH}$-type zinc-finger protein, $\mathrm{IbC} 3 \mathrm{H} 18$, functions as a nuclear transcriptional activator and enhances abiotic stress tolerance in sweet potato. New Phytol. 2019, 223, 1918-1936. [CrossRef]

42. Huang, L.; Cao, J.; Ye, W.; Liu, T.; Jiang, L.; Ye, Y. Transcriptional differences between the male-sterile mutant bcms and wild-type Brassica campestris ssp. chinensis reveal genes related to pollen development. Plant Biol. 2008, 10, 342-355. [CrossRef] [PubMed]

43. Huang, L.; Ye, W.-Z.; Liu, T.-T.; Cao, J.-S. Characterization of the male-sterile line bcajh97-01a/b and identification of candidate genes for genic male sterility in chinese cabbage-pak-choi. J. Am. Soc. Hortic. Sci. 2009, 134, 632-640. [CrossRef]

44. Shen, X.; Xu, L.; Liu, Y.; Dong, H.; Zhou, D.; Zhang, Y.; Lin, S.; Cao, J.; Huang, L. Comparative transcriptome analysis and ChIP-sequencing reveals stage-specific gene expression and regulation profiles associated with pollen wall formation in Brassica rapa. BMC Genom. 2019, 20, 264. [CrossRef]

45. Honys, D.; Twell, D. Transcriptome analysis of haploid male gametophyte development in Arabidopsis. Genome Biol. 2004, 5, R85. [CrossRef] [PubMed]

46. Jung, N.Y.; Bae, W.J.; Chang, J.H.; Kim, Y.C.; Cho, Y. Cloning, expression, purification, crystallization and preliminary X-ray diffraction analysis of the central zinc-binding domain of the human Mcm10 DNA-replication factor. Acta Crystallogr. F-Struct. Biol. Commun. 2008, 64, 495-497. [CrossRef] [PubMed]

47. Hwang, E.S.; Choi, A.; Ho, I.-C. Transcriptional regulation of GATA-3 by an intronic regulatory region and fetal liver zinc finger protein 1. J. Immunol. 2002, 169, 248-253. [CrossRef]

48. Wang, L.; Xu, Y.; Zhang, C.; Ma, Q.; Joo, S.-H.; Kim, S.-K.; Xu, Z.; Chong, K. OsLIC, a Novel CCCH-type zinc finger protein with transcription activation, mediates rice architecture via brassinosteroids signaling. PLoS ONE 2008, 3, e3521. [CrossRef]

49. Lai, W.S.; Kennington, E.A.; Blackshear, P.J. Tristetraprolin and its family members can promote the cell-free deadenylation of AU-rich element-containing mRNAs by poly (A) ribonuclease. Mol. Cell. Biol. 2003, 23, 3798-3812. [CrossRef]

50. Delaney, K.J.; Xu, R.; Zhang, J.; Li, Q.Q.; Yun, K.-Y.; Falcone, D.L.; Hunt, A.G. Calmodulin interacts with and regulates the RNA-binding activity of an Arabidopsis polyadenylation factor subunit. Plant Physiol. 2006, 140, 1507-1521. [CrossRef]

51. Birney, E.; Kumar, S.; Krainer, A.R. Analysis of the RNA-recognition motif and RS and RGG domains: Conservation in metazoan pre-mRNA splicing factors. Nucleic Acids Res. 1993, 21, 5803-5816. [CrossRef] [PubMed]

52. Callebaut, I.; Mornon, J.-P. LOTUS, a new domain associated with small RNA pathways in the germline. Bioinformatics 2010, 26, 1140-1144. [CrossRef] [PubMed]

53. Anantharaman, V.; Zhang, D.; Aravind, L. OST-HTH: A novel predicted RNA-binding domain. Biol. Direct 2010, 5, 13. [CrossRef] 
54. Twell, D. Male gametophyte development. In Plant Developmental Biology-Biotechnological Perspectives, 1st ed.; Pua, E.C., Davey, M.R., Eds.; Springer: Berlin/Heidelberg, Germany; New York, NY, USA, 2010; Volume 1, pp. 225-244.

55. Scott, R.J.; Spielman, M.; Dickinson, H.G. Stamen structure and function. Plant Cell 2004, 16, S46-S60. [CrossRef]

56. Twell, D.; Park, S.K.; Lalanne, E. Mechanisms of asymmetric division and cell fate determination in developing pollen. Trends Plant Sci. 1998, 3, 305-310. [CrossRef]

57. Twell, D.; Oh, S.; Honys, D. Pollen development, a genetic and transcriptomic view. In The Pollen Tube: A Cellular and Molecular Perspective, 1st ed.; Malho, R., Ed.; Springer: Berlin/Heidelberg, Germany; New York, NY, USA, 2006; Volume 3, pp. 15-45.

58. Borg, M.; Brownfield, L.; Khatab, H.; Sidorova, A.; Lingaya, M.; Twell, D. The R2R3 MYB transcription factor DUO1 activates a male germline-specific regulon essential for sperm cell differentiation in Arabidopsis. Plant Cell 2011, 23, 534-549. [CrossRef]

59. Borg, M.; Rutley, N.; Kagale, S.; Hamamura, Y.; Gherghinoiu, M.; Kumar, S.; Sari, U.; Esparza-Franco, M.A.; Sakamoto, W.; Rozwadowski, K.; et al. An EAR-Dependent Regulatory Module Promotes Male Germ Cell Division and Sperm Fertility in Arabidopsis. Plant Cell 2014, 26, 2098-2113. [CrossRef]

60. Sorensen, A.-M.; Kroeber, S.; Saedler, H. The ABNORMAL GAMETOPHYTES (AGM) gene product of Arabidopsis demonstrates a role in mitosis during gamete development. Plant Cell Physiol. 2004, 45, 905-913. [CrossRef]

61. Teng, C.; Dong, H.; Shi, L.; Deng, Y.; Mu, J.; Zhang, J.; Yang, X.; Zuo, J. Serine palmitoyltransferase, a key enzyme for de novo synthesis of sphingolipids, is essential for male gametophyte development in Arabidopsis. Plant Physiol. 2008, 146, 1322-1332. [CrossRef]

62. Persson, S.; Paredez, A.; Carroll, A.; Palsdottir, H.; Doblin, M.; Poindexter, P.; Khitrov, N.; Auer, M.; Somerville, C.R. Genetic evidence for three unique components in primary cell-wall cellulose synthase complexes in Arabidopsis. Proc. Natl. Acad. Sci. USA 2007, 104, 15566-15571. [CrossRef]

63. Golovkin, M.; Reddy, A.S.N. A calmodulin-binding protein from Arabidopsis has an essential role in pollen germination. Proc. Natl. Acad. Sci. USA 2003, 100, 10558-10563. [CrossRef] [PubMed]

64. Ju, Y.; Guo, L.; Cai, Q.; Ma, F.; Zhu, Q.-Y.; Zhang, Q.; Sodmergen. Arabidopsis JINGUBANG is a negative regulator of pollen germination that prevents pollination in moist environments. Plant Cell 2016, 28, 2131-2146. [CrossRef] [PubMed]

65. Yang, X.; Zhang, Q.; Zhao, K.; Luo, Q.; Bao, S.; Liu, H.; Men, S. The Arabidopsis GPR1 gene negatively affects pollen germination, pollen tube growth, and gametophyte senescence. Int. J. Mol. Sci. 2017, 18, 1303. [CrossRef] [PubMed]

66. Zhang, X.; Henriques, R.; Lin, S.-S.; Niu, Q.-W.; Chua, N.-H. Agrobacterium-mediated transformation of Arabidopsis thaliana using the floral dip method. Nat. Protoc. 2006, 2, 641-646. [CrossRef] [PubMed]

67. Mudunkothge, J.M.; Mudunkothge, B.A. The GUS reporter system in flower development studies. In Flower Development: Methods and Protocols, 1st ed.; Riechmann, J.L., Wellmer, F., Eds.; Springer: Berlin/Heidelberg, Germany; New York, NY, USA, 2014; Volume 1110, pp. 295-304.

68. Xiong, X.; Liu, W.; Jiang, J.; Xu, L.; Huang, L.; Cao, J. Efficient genome editing of Brassica campestris based on the CRISPR/Cas9 system. Mol. Genet. Genom. 2019, 294, 1251-1261. [CrossRef] [PubMed]

69. Alexander, M.P. Differential staining of aborted and non-aborted pollen. Stain Technol. 1969, 44, 117-122. [CrossRef]

70. Regan, S.M.; Moffatt, B.A. Cytochemical analysis of pollen development in wild-type Arabidopsis and a male-sterile mutant. Plant Cell 1990, 2, 877-889. [CrossRef]

71. Lin, S.; Dong, H.; Zhang, F.; Qiu, L.; Wang, F.; Cao, J.; Huang, L. BcMF8, a putative arabinogalactan protein-encoding gene, contributes to pollen wall development, aperture formation and pollen tube growth in Brassica campestris. Ann. Bot. 2014, 113, 777-788. [CrossRef]

72. Lin, S.; Yue, X.; Miao, Y.; Yu, Y.; Dong, H.; Huang, L.; Cao, J. The distinct functions of two classical arabinogalactan proteins BcMF8 and BcMF18 during pollen wall development in Brassica campestris. Plant J. 2018, 94, 60-76. [CrossRef]

(C) 2020 by the authors. Licensee MDPI, Basel, Switzerland. This article is an open access article distributed under the terms and conditions of the Creative Commons Attribution (CC BY) license (http://creativecommons.org/licenses/by/4.0/). 